\title{
Helmholtz Theorems, Gauge Transformations, General Covariance and the Empirical Meaning of Gauge Conditions
}

\author{
Andrew Chubykalo1, Augusto Espinoza1, Rolando Alvarado Flores ${ }^{2}$ \\ ${ }^{1}$ Unidad Academica de Fisica, Universidad Autónoma de Zacatecas, Zacatecas, Mexico \\ ${ }^{2}$ Centro de Estudios Multidisciplinarios, Universidad Autónoma de Zacatecas, Zacatecas, México \\ Email: achubykalo@yahoo.com.mx
}

Received 4 March 2016; accepted 28 May 2016; published 31 May 2016

Copyright (C) 2016 by authors and Scientific Research Publishing Inc.

This work is licensed under the Creative Commons Attribution International License (CC BY). http://creativecommons.org/licenses/by/4.0/

(c) (i) Open Access

\section{Abstract}

It is well known that the use of Helmholtz decomposition theorem for static vector fields $C: \mathbf{R}^{3} \rightarrow \mathbf{R}^{3}$, when applied to the time dependent vector fields $E: R^{4} \rightarrow R^{3}, B: R^{4} \rightarrow R^{3}$ which represent the electromagnetic field, allows us to obtain instantaneous-like solutions all along $\mathbf{R}^{3}$. For this reason, some people thought (see e.g. [1] and references therein) that the Helmholtz theorem cannot be applied to time dependent vector fields and some modification is wanted in order to get the retarded solutions. However, the use of the Helmholtz theorem for static vector fields is correct even for time dependent vector fields (see, e.g. [2]), so a relation between the solutions was required, in such a way that a retarded solution can be transformed in an instantaneous one, and conversely. On this paper we want to suggest, following most of the time the mathematical formalism of Woodside in [3], that: 1) there are many Helmholtz decompositions, all equally consistent, 2) each one is naturally related to a space-time structure, 3) when we use the Helmholtz decomposition for the electromagnetic potentials it is equivalent to a gauge transformation, 4) there is a natural methodological criterion for choosing the gauge according to the structure postulated for a global spacetime, 5) the Helmholtz decomposition is the manifestation at the level of the fields that a gauge is involved. So, when we relate the retarded solution to the instantaneous one what we do is to change the gauge and the space-time. And, if the Helmholtz decompositions are related to a space-time structure, and are equivalent to gauge transformations, each gauge transformation is natural for a specific space-time. In this way, a Helmholtz decomposition for Euclidean space is equivalent to the Coulomb gauge and a Helmholtz decomposition for the Minkowski space is equivalent to the Lorenz gauge. This leads us to consider that the theories defined by different gauges may be mathematically equivalent, because they can be related by means of a gauge transformation, but they are not empirically equivalent, because they have quite different observational consequences due to the different space-time structure involved. 


\section{Keywords}

\section{Helmholtz Theorem, Gauge Transformations, Space-Time Transformations, Symmetries of Differential Equations, Underdetermination of Systems of Differential Equations, Natural Covariance}

\section{Introduction}

Starting directly from Maxwell equations for the pair of time dependent vector fields that represent the electromagnetic field, $\boldsymbol{E}$ and $\boldsymbol{B}$, it is possible to deduce a pair of second order, inhomogeneous, partial differential equations of apparently hyperbolic type given by:

$$
\left.\begin{array}{l}
\left(\Delta-\frac{1}{c^{2}} \frac{\partial^{2}}{\partial t^{2}}\right) \boldsymbol{E}(\boldsymbol{x}, t)=4 \pi \nabla \varrho+\frac{4 \pi}{c^{2}} \frac{\partial \boldsymbol{J}}{\partial t} \\
\left(\Delta-\frac{1}{c^{2}} \frac{\partial^{2}}{\partial t^{2}}\right) \boldsymbol{B}(\boldsymbol{x}, t)=-\frac{4 \pi}{c} \nabla \times \boldsymbol{J}
\end{array}\right\}
$$

We use common notation for the Laplace operator $\Delta$, the gradient operator $\nabla$, and the matter fields, $\varrho$ and $\boldsymbol{J}$, which stand for charge density and current density while $c$ is the speed of light. We use the Gaussian system of units. If the time dependent vector fields that represent the electromagnetic field vanishes at infinity, and the sources are localized, we can use classical retarded solutions for this kind of inhomogeneous D'Alembert equations, so it is possible to understand the electromagnetic field at a space-time point $\langle\boldsymbol{x}, t\rangle$ as the result of charges and currents located at a compact domain $D$ of space at a time $t^{\prime}=t-\frac{\left|\boldsymbol{x}-\boldsymbol{x}^{\prime}\right|}{c}$, where $\boldsymbol{x}^{\prime} \in D$. So the electromagnetic field is a perturbation with finite propagation speed that respects causality, because its effects are always after the cause (this can be formulated as Huygens' principle, see [4]). It seems that there is no room for instantaneous solutions. We can obtain instantaneous solutions for Maxwell equations using the Helmholtz theorem (HT) (see e.g. [5] and [6]) and for this reason it was believed by some people that something must go wrong using it (notably Heras in [3]), and proposed new forms of the Helmholtz decomposition, such that the retarded solutions can be obtained out of it (see e.g. [7] [8] and [3]). However, what all these discussions showed is that there are many ways to obtain a Helmholtz-like decomposition and as many solutions to the Maxwell equations; but the relationship among all these solutions remained obscure. In this paper we are going to prove that each the Helmholtz decomposition, when applied to the potentials, is equivalent to a gauge transformation, and that each gauge transformation is naturally related to a space-time, obtaining a methodological criterion for the choice of gauge. So, we can understand the application of the Helmholtz theorem to the field strengths as a gauge transformation also, that relates solutions in different gauges. We start our treatment at the level of the electromagnetic field vectors, in Section 3, represented by continuous and $n$ times differentiable-hence of the class $C^{n}$, time dependent vector functions $\boldsymbol{E}: \mathbf{R}^{4} \rightarrow \mathbf{R}^{3}, \quad \boldsymbol{B}: \mathbf{R}^{4} \rightarrow \mathbf{R}^{3}$ vanishing at infinity with all its derivatives. We are going to display three Helmholtz decompositions adapted for the three canonical forms of second order partial differential equations and we will show that jointly solving the Maxwell's equations with a Helmholtz decomposition we will obtain a known representation for the fields in terms of well-defined functions that in Section 4 we are going to identify with the potentials in a fixed gauge. Of course, the cases for the D'Alembert operator and the Laplace operator are well-known. At this level there is no question about the gauge, and the relation among the solutions becomes a matter of applying wisely the Helmholtz decomposition as we will show. If we want to go deeper into the structure of the Helmholtz decompositions we need the gauge potentials $\langle\boldsymbol{A}, \varphi\rangle$ which we suppose of class $C^{n}$ too, vanishing at infinity with all its derivatives. This is the topic of Section 4. We shall prove that each the Helmholtz decomposition of the electromagnetic potentials is equivalent to a gauge transformation, and because the solutions of the Maxwell equations for the potentials at each different gauge give rise to the corresponding solutions for the field strengths, we can understand that the Helmholtz decomposition that relates retarded and instantaneous solutions is a gauge transformation. So, even the instantaneous solutions are correct solutions of the Maxwell equations that result from the 
use of a particular Helmholtz decomposition. We can obtain out of them the retarded ones changing the gauge, i.e., using some other Helmholtz decomposition for the field strengths or for the potentials. Even more, we shall see that the Coulomb gauge is related to the Euclidean space, while the Lorenz gauge is related to the Minkowski space, with the Helmholtz decompositions_or gauge transformations_-functioning as a bridge between them. This is enough, at least in our humble opinion, to support the idea that each Helmholtz theorem reflects a characteristic of the space-time geometry. So, because the Helmholtz decomposition is related to a gauge in a unique way the space-time form is related to the choice of gauge. But if this is so, we conclude that the Maxwell electromagnetic field equations, its theoretical and operational concepts and the whole of its experimental confirmations are not enough to make the theory complete, and for this reason, and this reason alone, the instantaneous and the retarded solutions are possible solutions of the field equations. Obviously, there are ways to complete the theory, like the Special Theory of Relativity (STR), but this is a sort of a posteriori completeness process depending on experiment rather than on theoretical grounds. In Section 5, we shall try to outline a method to obtain the space-time out of the Maxwell equations, the gauge and the symmetries of the differential equations defined by the gauge, and we shall explain in more detail all we have announced here by making a comparison with standard procedures that, in our opinion, are not completely understood. Section 2 is a mathematical preliminary where we put all the mathematical properties and identities that we shall use freely all along the paper, hence is a section for reference that can be skipped if desired.

\section{Mathematical Preliminary}

In this paper we shall use the Green's function formalism, so we can avoid the retardation symbol and its awkward expressions. Each of our Green's functions is going to be one of the following set:

$$
\left.\begin{array}{l}
\left(\Delta-\frac{1}{c^{2}} \frac{\partial^{2}}{\partial t^{2}}\right) G_{d}\left(\boldsymbol{x}, t ; \boldsymbol{x}^{\prime}, t^{\prime}\right)=-\frac{4 \pi}{c} \delta\left(\boldsymbol{x}-\boldsymbol{x}^{\prime}\right) \delta\left(t-t^{\prime}\right) \\
\Delta G_{l}\left(\boldsymbol{x}, t ; \boldsymbol{x}^{\prime}, t^{\prime}\right)=-\frac{4 \pi}{c} \delta\left(\boldsymbol{x}-\boldsymbol{x}^{\prime}\right) \delta\left(t-t^{\prime}\right) \\
\left(\Delta-D \frac{\partial}{\partial t}\right) G_{f}\left(\boldsymbol{x}, t ; \boldsymbol{x}^{\prime}, t^{\prime}\right)=-\frac{4 \pi}{c} \delta\left(\boldsymbol{x}-\boldsymbol{x}^{\prime}\right) \delta\left(t-t^{\prime}\right)
\end{array}\right\}
$$

Here $D$ is just a dimensional constant. We can solve these equations by means of spectral decomposition. If so, we must go into the complex plane to define a path in order to obtain the Green's function by encircling its singularities. Such a process we shall suppose done, so we have the following results:

$$
\left.\begin{array}{l}
G_{d}\left(\boldsymbol{x}, t ; \boldsymbol{x}^{\prime}, t^{\prime}\right)=\frac{1}{4 \pi} \frac{\delta\left(t-\left(t^{\prime}-\frac{\boldsymbol{x}-\boldsymbol{x}^{\prime}}{c}\right)\right)}{\left|\boldsymbol{x}-\boldsymbol{x}^{\prime}\right|} \\
G_{l}\left(\boldsymbol{x}, t ; \boldsymbol{x}^{\prime}, t^{\prime}\right)=\frac{1}{4 \pi} \frac{\delta\left(t-t^{\prime}\right)}{\left|\boldsymbol{x}-\boldsymbol{x}^{\prime}\right|} \\
G_{f}\left(\boldsymbol{x}, t ; \boldsymbol{x}^{\prime}, t^{\prime}\right)=\frac{1}{\left(4 \pi D\left(t-t^{\prime}\right)\right)^{\frac{3}{2}}} \mathrm{e}^{-\frac{\left|\boldsymbol{x}-\boldsymbol{x}^{\prime}\right|^{2}}{4 \pi D\left(t-t^{\prime}\right)}}
\end{array}\right\}
$$

The following properties of the Green's functions will be very useful:

$$
\left.\begin{array}{l}
\lim _{t \rightarrow \pm \infty} G\left(\boldsymbol{x}, t ; \boldsymbol{x}^{\prime}, t^{\prime}\right)=0, \lim _{t \rightarrow \pm \infty} \frac{\partial G\left(\boldsymbol{x}, t ; \boldsymbol{x}^{\prime}, t^{\prime}\right)}{\partial t}=0 \\
\frac{\partial G\left(\boldsymbol{x}, t ; \boldsymbol{x}^{\prime}, t^{\prime}\right)}{\partial t}=-\frac{\partial G\left(\boldsymbol{x}, t ; \boldsymbol{x}^{\prime}, t^{\prime}\right)}{\partial t^{\prime}} \\
\nabla G\left(\boldsymbol{x}, t ; \boldsymbol{x}^{\prime}, t^{\prime}\right)=-\nabla^{\prime} G\left(\boldsymbol{x}, t ; \boldsymbol{x}^{\prime}, t^{\prime}\right)
\end{array}\right\} .
$$


Also we shall use along the development, the following identities, valid for any Green's function and time dependent vector function $\left(x^{\prime}, t^{\prime}\right)$ :

$$
\begin{aligned}
& G\left(\boldsymbol{x}, t ; \boldsymbol{x}^{\prime}, t^{\prime}\right) \nabla^{\prime}\left(\nabla^{\prime} \cdot \boldsymbol{C}\left(\boldsymbol{x}^{\prime}, t^{\prime}\right)\right) \\
& =\nabla^{\prime}\left(G\left(\boldsymbol{x}, t ; \boldsymbol{x}^{\prime}, t^{\prime}\right) \nabla^{\prime} \cdot \boldsymbol{C}\left(\boldsymbol{x}^{\prime}, t^{\prime}\right)\right)-\nabla^{\prime} \cdot \boldsymbol{C}\left(\boldsymbol{x}^{\prime}, t^{\prime}\right) \nabla^{\prime} G\left(\boldsymbol{x}, t ; \boldsymbol{x}^{\prime}, t^{\prime}\right), \\
& G\left(\boldsymbol{x}, t ; \boldsymbol{x}^{\prime}, t^{\prime}\right) \nabla^{\prime} \times \nabla^{\prime} \times \boldsymbol{C}\left(\boldsymbol{x}^{\prime}, t^{\prime}\right) \\
& =\nabla^{\prime} \times\left(G\left(\boldsymbol{x}, t ; \boldsymbol{x}^{\prime}, t^{\prime}\right) \nabla^{\prime} \times \nabla^{\prime} \times \boldsymbol{C}\left(\boldsymbol{x}^{\prime}, \boldsymbol{t}^{\prime}\right)\right)-\nabla G\left(\boldsymbol{x}, t ; \boldsymbol{x}^{\prime}, t^{\prime}\right) \nabla^{\prime} \times \nabla^{\prime} \times \boldsymbol{C}\left(\boldsymbol{x}^{\prime}, t^{\prime}\right), \\
& G\left(\boldsymbol{x}, t ; \boldsymbol{x}^{\prime}, t^{\prime}\right) \frac{\partial^{2} \boldsymbol{C}\left(\boldsymbol{x}^{\prime}, t^{\prime}\right)}{\partial t^{\prime 2}} \\
& =\frac{\partial}{\partial t^{\prime}}\left(G\left(\boldsymbol{x}, t ; \boldsymbol{x}^{\prime}, t^{\prime}\right) \frac{\partial \boldsymbol{C}\left(\boldsymbol{x}^{\prime}, t^{\prime}\right)}{\partial t^{\prime}}\right)-\frac{\partial G\left(\boldsymbol{x}, t ; \boldsymbol{x}^{\prime}, t^{\prime}\right)}{\partial t^{\prime}} \frac{\partial \boldsymbol{C}\left(\boldsymbol{x}^{\prime}, t^{\prime}\right)}{\partial t^{\prime}} .
\end{aligned}
$$

All the components of our time dependent vector functions are going to be tempered functions, that is, of the Schwarz class. The definition runs as follows:

$$
S^{k}=\left\{f \in C^{k}|\sup | x^{m} \frac{\partial^{n} f}{\partial x^{n}} \mid \leq c_{n, m}<\infty, n, m \in \mathbf{N}\right\} .
$$

This is enough for all boundary terms to vanish and for the Riemann integrability of the functions. Sometimes, of course, the relevant variable of our tempered functions will be the spherical variable $r$, or the vector norm. We shall suppose that all vector norms are Schwarz class also. So, when we integrate along all the space or the time we obtain the following integral identities:

$$
\begin{gathered}
\int G\left(\boldsymbol{x}, t ; \boldsymbol{x}^{\prime}, t^{\prime}\right) \nabla^{\prime}\left(\nabla^{\prime} \cdot \boldsymbol{C}\left(\boldsymbol{x}^{\prime}, t^{\prime}\right)\right) \mathrm{d} V^{\prime} \mathrm{d} t^{\prime}=-\int \nabla^{\prime} \cdot \boldsymbol{C}\left(\boldsymbol{x}^{\prime}, t^{\prime}\right) \nabla^{\prime} G\left(\boldsymbol{x}, t ; \boldsymbol{x}^{\prime}, t^{\prime}\right) \mathrm{d} V^{\prime} \mathrm{d} t^{\prime}, \\
\int G\left(\boldsymbol{x}, t ; \boldsymbol{x}^{\prime}, t^{\prime}\right) \nabla^{\prime} \times \nabla^{\prime} \times \boldsymbol{C}\left(\boldsymbol{x}^{\prime}, t^{\prime}\right) \mathrm{d} V^{\prime} \mathrm{d} t^{\prime}=-\int \nabla^{\prime} \cdot \boldsymbol{C}\left(\boldsymbol{x}^{\prime}, t^{\prime}\right) \nabla^{\prime} G\left(\boldsymbol{x}, t ; \boldsymbol{x}^{\prime}, t^{\prime}\right) \mathrm{d} V^{\prime} \mathrm{d} t^{\prime}, \\
\int G\left(\boldsymbol{x}, t ; \boldsymbol{x}^{\prime}, t^{\prime}\right) \frac{\partial^{2} \boldsymbol{C}\left(\boldsymbol{x}^{\prime}, t^{\prime}\right)}{\partial t^{\prime 2}} \mathrm{~d} V^{\prime} \mathrm{d} t^{\prime}=-\int \frac{\partial G\left(\boldsymbol{x}, t ; \boldsymbol{x}^{\prime}, t^{\prime}\right)}{\partial t^{\prime}} \frac{\partial \boldsymbol{C}\left(\boldsymbol{x}^{\prime}, t^{\prime}\right)}{\partial t^{\prime}} \mathrm{d} V^{\prime} \mathrm{d} t^{\prime} .
\end{gathered}
$$

Therefore we discard all surface terms obtained using Gauss theorem avoiding questions of uniqueness, so relevant for the general theorems of [3]. One can also fix the ambiguities arising from surface terms with well-defined boundary value problems, but we shall not pursue that subject because we are going to work in the infinite domain. We shall use a Minkowski space-time $M=\left\langle\eta_{\mu \sigma}, \mathbf{R}^{4}\right\rangle$ defined by the metric $\eta_{\mu \sigma}$ given in any orthogonal frame by its diagonal terms $(-1,1,1,1)$ which we can use to raise and lower indexes to identify covariant and contravariant vectors; i.e., the tangent space of our Minkowski space-time and its dual one.

\section{Helmholtz Theorems and Maxwell Equations for the Electromagnetic Field}

Maxwell equations for the electromagnetic field strengths $\boldsymbol{E}$ and $\boldsymbol{B}$ are in the Gaussian system of units:

$$
\left.\begin{array}{l}
\nabla \cdot \boldsymbol{E}=4 \pi \varrho \\
\nabla \times \boldsymbol{E}=-\frac{1}{c} \frac{\partial \boldsymbol{B}}{\partial t} \\
\nabla \cdot \boldsymbol{B}=0 \\
\nabla \times \boldsymbol{B}=\frac{1}{c} \frac{\partial \boldsymbol{E}}{\partial t}+\frac{4 \pi}{c} \boldsymbol{J}
\end{array}\right\}
$$

There are, as has been remarked by Woodside [3], three related theorems that deserve the name of "Helmholtz". The first one is as follows:

\section{Helmholtz Theorem A:}

A static vector field $\mathbf{C}$ in an Euclidean 3-space is uniquely determined all along a bounded region $G$ and a closed surface $S$ bounding $G$, once we know its rotational $\nabla \times C$ and divergence $\nabla \cdot C$ at every point of $G$ 
and its normal components on $S$.

For short, we shall call a continuous and differentiable vector field with its derivatives up to order $k$ vanishing at infinity a $S^{k}$ vector field.

The second and third ones are HT's for infinite and finite domains in a Euclidean 3-space:

\section{Helmholtz Theorem B:}

A $S^{k}, k \geq 1$, static vector field defined over all of a Euclidean 3-space may be uniquely represented as a sum of an irrotational and a solenoidal part, up to an additive constant.

\section{Helmholtz Theorem C}

A $S^{k}, k \geq 1$, static vector field defined over all of a compact subset $G$ of a Euclidean 3-space, where over a surface bounding $G$ its normal and tangential components are known, may be uniquely represented as a sum of an irrotational and a solenoidal part, up to an additive constant.

For proofs and comments see [3]. In this paper all the theorems will be stated for the infinite domain, whose prototypical enunciation is theorem $\mathrm{B}$, and all the conditions given in the theorems will be sufficient conditions. We can slightly modify Helmholtz theorem B for time dependent vector field $\boldsymbol{C}$ with tempered components, so they can be represented as follows:

$$
\begin{aligned}
\boldsymbol{C}(\boldsymbol{x}, t)= & \nabla \times\left(\int G_{l}\left(\boldsymbol{x}, t ; \boldsymbol{x}^{\prime}, t^{\prime}\right) \nabla^{\prime} \times \mathbf{C}\left(\boldsymbol{x}^{\prime}, t^{\prime}\right) \mathrm{d} V^{\prime} \mathrm{d} t^{\prime}\right) \\
& +\nabla \cdot\left(\int G_{l}\left(\boldsymbol{x}, t ; \boldsymbol{x}^{\prime}, t^{\prime}\right) \nabla^{\prime} \cdot \boldsymbol{C}\left(\boldsymbol{x}^{\prime}, t^{\prime}\right) \mathrm{d} V^{\prime} \mathrm{d} t^{\prime}\right) .
\end{aligned}
$$

From this representation we can prove Helmholtz theorem A [3] but it is clear that not one of these theorems can be applied directly to the vector fields $\boldsymbol{E}$ and $\boldsymbol{B}$ because the rotational of each one of them involves, again, the fields, and what we want is a determination involving the current and charge densities because these are the sources of the fields. The procedure to solve the Maxwell equations for the fields lies in the postulation of a Helmholtz decomposition for each field that is substituted in (2) for separating the irrotational and solenoidal components of each time dependent vector. In this way we shall get differential equations for these components that can be solved providing us with a representation of the fields in terms of the current and charge densities. We shall call this result: "Helmholtz-Maxwell Elliptic Theorem" (HMET).

\section{Theorem 1 (Helmholtz-Maxwell Elliptic Theorem)}

If three $S^{k}, k \geq 1$, time dependent vector fields $\boldsymbol{E}, \boldsymbol{B}$ and $\boldsymbol{J}$ and a scalar time dependent field $\rho$ are such that satisfy the following conditions:

1) They can be decomposed as $=\boldsymbol{E}_{s}+\boldsymbol{E}_{i}, \boldsymbol{B}=\boldsymbol{B}_{s}, \boldsymbol{J}=\boldsymbol{J}_{s}+\boldsymbol{J}_{I}$ with $\nabla \cdot \boldsymbol{E}_{s}=0, \nabla \cdot \boldsymbol{B}_{s}=0, \nabla \times \boldsymbol{E}_{i}=0$, $\nabla \cdot \boldsymbol{J}_{s}=0, \nabla \times \boldsymbol{J}_{i}=0$

2) They satisfy Maxwell Equation (2)

3) $\rho \in S^{k}, k>1$

Then the electromagnetic field vectors admit the following representation:

$$
\left.\begin{array}{l}
\boldsymbol{E}(\boldsymbol{x}, t)=-\nabla \int G_{l}\left(\boldsymbol{x}, t ; \boldsymbol{x}^{\prime}, t^{\prime}\right) \varrho\left(\boldsymbol{x}^{\prime}, t^{\prime}\right) \mathrm{d} V^{\prime} \mathrm{d} t^{\prime}+\frac{4 \pi}{c^{2}} \frac{\partial}{\partial t} \int G_{d}\left(\boldsymbol{x}, t ; \boldsymbol{x}^{\prime}, t^{\prime}\right) \boldsymbol{J}_{s}\left(\boldsymbol{x}^{\prime}, t^{\prime}\right) \mathrm{d} V^{\prime} \mathrm{d} t^{\prime} \\
\boldsymbol{B}(\boldsymbol{x}, t)=\frac{4 \pi}{c} \int G_{d}\left(\boldsymbol{x}, t ; \boldsymbol{x}^{\prime}, t^{\prime}\right) \nabla^{\prime} \times \boldsymbol{J}_{s}\left(\boldsymbol{x}^{\prime}, t^{\prime}\right) \mathrm{d} V^{\prime} \mathrm{d} t
\end{array}\right\}
$$

where $d V^{\prime}$ is the spatial volume element in the Cartesian coordinates $\boldsymbol{x}^{\prime}$.

Proof: the conditions are sufficient conditions hence the proof is straigthforward. We must put the decompositions in Maxwell Equation (2) to separate rotational and solenoidal components obtaining sets of differential equations we can solve. We get the following sets of equations:

$$
\left.\begin{array}{l}
\nabla \cdot \boldsymbol{E}_{s}=0 \\
\nabla \times \boldsymbol{E}_{s}=-\frac{1}{c} \frac{\partial \boldsymbol{B}_{s}}{\partial t} \\
\nabla \cdot \boldsymbol{B}_{s}=0 \\
\nabla \times \boldsymbol{B}_{s}=-\frac{1}{c} \frac{\partial \boldsymbol{E}_{s}}{\partial t}+\frac{4 \pi}{c} \boldsymbol{J}_{s}
\end{array}\right\}
$$




$$
\left.\begin{array}{l}
\nabla \cdot \boldsymbol{E}_{i}=4 \pi \varrho \\
\nabla \times \boldsymbol{E}_{i}=0 \\
\frac{1}{c} \frac{\partial \boldsymbol{E}_{i}}{\partial t}=-\frac{4 \pi}{c} \boldsymbol{J}_{i}
\end{array}\right\}
$$

Now, when we solve (4), under the stated assumptions of differentiability, we obtain a Dirichlet boundary value problem for the Poisson equation in the infinite domain for the irrotational component of the electric field, i.e.:

$$
\begin{gathered}
\Delta \boldsymbol{E}_{i}=-4 \pi \nabla \varrho \\
\lim _{|x| \rightarrow \infty} \boldsymbol{E}_{i}(\boldsymbol{x}, t) \rightarrow 0
\end{gathered}
$$

Whose solution exists and is given HMET1. Even more, because of the stated condition on $\rho$ the solution is everywhere uniformly continuous and differentiable (see [9] p. 246). Hence we get the instantaneous component. When we solve (3) what we obtain is the following problem:

$$
\begin{gathered}
\left(\Delta-\frac{1}{c^{2}} \frac{\partial^{2}}{\partial t^{2}}\right) \boldsymbol{E}_{S}(\boldsymbol{x}, t)=\frac{4 \pi}{c^{2}} \frac{\partial \boldsymbol{J}_{S}}{\partial t} \\
\lim _{|x| \rightarrow \infty} \boldsymbol{E}_{S}(\boldsymbol{x}, t) \rightarrow 0
\end{gathered}
$$

And another similar problem for the magnetic field. These sort of boundary value problems for hyperbolic equations require initial non-characteristic conditions for uniqueness of solutions, however under the stated assumptions the functions given in HMET1 solve the problem and we obtain the retarded components. QED.

As we have been remarked we are interested in sufficient conditions, i.e. given the set of conditions a solution can be obtained, but we are not interested in necessary conditions, i.e. given a solution that solution satisfies the stated conditions. This is no always the case, because we can obtain different solutions for finite domains, i.e. where the condition of vanishing at infinite is not satisfied. e.g. any solution of the Poisson equation for the sphere.

Indeed the irrotational component of the HMET1 is just like the same component of the usual Helmholz theorem, and is the source of many discussions; while the solenoidal component is quite different, first of all because it is retarded. The Equations ((3), (4)) were obtained by Rohrlich in his book [10] however he made no effort to solve and interpret them; most of the cited book is about the retarded solutions (and the retarded action at a distance). But in his paper [5] he proved that the HMET1 is consistent with Equation (1). Indeed his words were: “... this nonlocal relation of the longitudinal field to the total field is exactly responsible for turning the retarded field $\boldsymbol{E}$ due to $\varrho \ldots$... into an instantaneous (action-at-a-distance) field...”. Hence the electric field is not totally retarded, and the charge density produces a static like-instantaneous-field together with the retarded component due to the time variation of the solenoidal component of the current density. The magnetic field is clearly produced by the rotational of the current density, and no problem arises from it. But in [1] (the reply to [5]) J. Heras claimed that: “... Rohrlich's conclusion is based on an inconsistent mathematical procedure, which is due to the common misconception that the standard Helmholtz theorem... can be applied to retarded vector fields.”. However in his paper [2] Woodside explained us why the Helmholtz theorem can be applied to time dependent vector fields: “...first observe that the delta function integral property...holds even for well-behaved time-varying functions $\boldsymbol{F}(x, y, z, t)$ because the space and time variables are independent, and consequently space and time integrations are performed separately". In note (3) of paper [1] Heras told us that: "Helmholtz theorem can consistently applied to time dependent vector fields only when an instantaneous propagation for the fields is assumed". This statement and Woodside's assumptions are enough to suggest that the usual Helmholtz theorem can be used in Euclidean space-times with a meaningful relation of simultaneity. This is an indication that the Helmholtz theorem carries a relation with the space-time structure because time and space are independent on certain space-times only. Not being the case in a Minkowski space-time with metric $|\boldsymbol{x}|^{2}-c^{2} t^{2}$ where space and time are related. Now let us explain why a retarded solution becomes instantaneous. For Rohrlich the explanation for the "conspiracy" that turns the retarded solution into the instantaneous one lies in the nonlocal 
relation relating the time dependent vector to its longitudinal and transversal components, a relation arising directly from Helmholtz decomposition. However, such explanation is insufficient. In fact, we believe that the transformation from the retarded solution to the instantaneous one results directly from the Helmholtz decomposition of the current density and the Equation (4). To see it let us write down the usual retarded solution arising from Equation (1):

$$
\int G_{d}\left(\boldsymbol{x}, t ; \boldsymbol{x}^{\prime}, t^{\prime}\right)\left(\nabla^{\prime} \varrho\left(\boldsymbol{x}^{\prime}, t^{\prime}\right)+\frac{1}{c^{2}} \frac{\partial \boldsymbol{J}\left(\boldsymbol{x}^{\prime}, t^{\prime}\right)}{\partial t^{\prime}}\right) \mathrm{d} V^{\prime} \mathrm{d} t^{\prime}
$$

If we apply the conditions of the HMET, i.e., we separate the current density as $\boldsymbol{J}_{=} \boldsymbol{J}_{s}+\boldsymbol{J}_{i}$ then the irrotational component of the retarded solution becomes:

$$
\int G_{d}\left(\boldsymbol{x}, t ; \boldsymbol{x}^{\prime}, t^{\prime}\right)\left(\nabla^{\prime} \varrho\left(\boldsymbol{x}^{\prime}, t^{\prime}\right)+\frac{1}{c^{2}} \frac{\partial \boldsymbol{J}_{i}\left(\boldsymbol{x}^{\prime}, t^{\prime}\right)}{\partial t^{\prime}}\right) \mathrm{d} V^{\prime} \mathrm{d} t^{\prime} .
$$

From the Equation (4) without much ado we get:

$$
\int G_{d}\left(\boldsymbol{x}, t ; \boldsymbol{x}^{\prime}, t^{\prime}\right)\left(\frac{1}{4 \pi} \nabla^{\prime}\left(\nabla^{\prime} \cdot \boldsymbol{E}_{i}\left(\boldsymbol{x}^{\prime}, t^{\prime}\right)\right)-\frac{1}{4 \pi c^{2}} \frac{\partial^{2} \boldsymbol{E}_{i}\left(\boldsymbol{x}^{\prime}, t^{\prime}\right)}{\partial t^{\prime 2}}\right) \mathrm{d} V^{\prime} \mathrm{d} t^{\prime} .
$$

Hence, using the known vector identity:

$$
\nabla \times \nabla \times \boldsymbol{A}=\nabla(\nabla \cdot \boldsymbol{A})-\Delta \boldsymbol{A}
$$

For the irrotational component of the electric field we obtain:

$$
\int G_{d}\left(x, t ; x^{\prime}, t^{\prime}\right)\left(\frac{1}{4 \pi} \Delta^{\prime} \boldsymbol{E}_{i}\left(\boldsymbol{x}^{\prime}, t^{\prime}\right)-\frac{1}{4 \pi c^{2}} \frac{\partial^{2} \boldsymbol{E}_{i}\left(\boldsymbol{x}^{\prime}, t^{\prime}\right)}{\partial t^{\prime 2}}\right) \mathrm{d} V^{\prime} \mathrm{d} t^{\prime}=\boldsymbol{E}_{i}(\boldsymbol{x}, t),
$$

where an integration by parts was used. That is, the retarded solution becomes the instantaneous one, which is, of course, the result obtained by Rohrlich using potentials. But we can see an explanation in the fact that we have applied the sufficient conditions for the validity of the HMET: the separation of the time dependent vectors in irrotational and solenoidal components and that all these satisfy Maxwell equations. So Heras' claim is right, indeed, it is the application of the HMET the reason for the reduction of the retarded to the instantaneous solution. Hence, a new Helmholtz decomposition is required to avoid the instantaneous solution. This is what Heras proposed and proved to be a consistent and rich procedure in several papers by deducing Jefimenko's formulas with magnetic monopoles [7], or for dipoles in motion [11] obtaining generalizations of the Biot-Savart and Coulomb's laws [12] or alternate derivation of the Lienard-Wiechert fields. This important result we may call "Helmholtz Hyperbolic Theorem". It runs as follows:

Theorem 2 (Helmholtz Hyperbolic Theorem):

A $S^{k}, k \geq 1$, time dependent vector field defined over all of a Minkowski space-time may be uniquely represented as a sum of an irrotational, a solenoidal and a time part, up to an additive constant.

Comment: Again the proof is straightforward and can be seen in [3]. However Heras in [7] and [12] proved it without specifying the geometry of space-time, but certainly, it must have been for a Minkowski space-time because his objective was to avoid instantaneous solutions, and for this it seems unavoidable the use of a Minkowski space-time geometry because by design in this space-time signals cannot propagate at superluminous speeds. So we can follow the steps of this proof using 3-dimensional Gibbs'notation for vectors. See also [2]. Of course, this is somehow an anachronism.

Proof: Everything starts out from the identity:

$$
\left(\Delta-\frac{1}{c^{2}} \frac{\partial^{2}}{\partial t^{2}}\right) \boldsymbol{C}=\nabla(\nabla \cdot \boldsymbol{C})-\nabla \times \nabla \times \boldsymbol{C}-\frac{1}{c^{2}} \frac{\partial^{2} \boldsymbol{C}}{\partial t^{2}} .
$$

Next we represent the time dependent vector $\boldsymbol{C}$ as:

$$
C(x, t)=\int \delta\left(x-x^{\prime}\right) \delta\left(t-t^{\prime}\right) C\left(x^{\prime}, t^{\prime}\right) \mathrm{d} V^{\prime} \mathrm{d} t^{\prime} .
$$


Introducing the Green's function $G_{d}\left(\boldsymbol{x}, t ; \boldsymbol{x}^{\prime}, \boldsymbol{t}^{\prime}\right)$ we can write down:

$$
\boldsymbol{C}(\boldsymbol{x}, t)=-\frac{c}{4 \pi} \int\left(\Delta^{\prime}-\frac{1}{c^{2}} \frac{\partial^{2}}{\partial t^{\prime 2}}\right) G_{d}\left(\boldsymbol{x}, t ; \boldsymbol{x}^{\prime}, t^{\prime}\right) \boldsymbol{C}\left(\boldsymbol{x}^{\prime}, t^{\prime}\right) \mathrm{d} V^{\prime} \mathrm{d} t^{\prime} .
$$

An integration by parts produces:

$$
\boldsymbol{C}(\boldsymbol{x}, t)=-\frac{c}{4 \pi} \int G_{d}\left(\boldsymbol{x}, t ; \boldsymbol{x}^{\prime}, t^{\prime}\right)\left(\Delta^{\prime}-\frac{1}{c^{2}} \frac{\partial^{2}}{\partial t^{\prime 2}}\right) \boldsymbol{C}\left(\boldsymbol{x}^{\prime}, t^{\prime}\right) \mathrm{d} V^{\prime} \mathrm{d} t^{\prime} .
$$

Using (10) we get:

$$
\boldsymbol{C}(\boldsymbol{x}, t)=-\frac{c}{4 \pi} \int G_{d}\left(\boldsymbol{x}, t ; \boldsymbol{x}^{\prime}, \boldsymbol{t}^{\prime}\right) \cdot\left(\nabla^{\prime}\left(\nabla^{\prime} \cdot \boldsymbol{C}\left(\boldsymbol{x}^{\prime}, t^{\prime}\right)\right)-\nabla^{\prime} \times \boldsymbol{C}\left(\boldsymbol{x}^{\prime}, \boldsymbol{t}^{\prime}\right)-\frac{1}{c^{2}} \frac{\partial^{2} \boldsymbol{C}\left(\boldsymbol{x}^{\prime}, t^{\prime}\right)}{\partial t^{\prime 2}}\right) \mathrm{d} V^{\prime} \mathrm{d} t^{\prime} .
$$

It is almost done, now we must use the identities (G2-3-4-5) to finally obtain:

$$
C(x, t)=C_{1}(x, t)+C_{2}(x, t)+C_{3}(x, t)
$$

with the following definitions:

$$
\begin{aligned}
& C_{1}(x, t)=-\frac{c}{4 \pi} \nabla \int G_{d}\left(x, t ; x^{\prime}, t^{\prime}\right) \nabla^{\prime} \cdot \boldsymbol{C}\left(\boldsymbol{x}^{\prime}, t^{\prime}\right) \mathrm{d} V^{\prime} \mathrm{d} t^{\prime}, \\
& \boldsymbol{C}_{2}(\boldsymbol{x}, t)=\frac{c}{4 \pi} \nabla \times \int G_{d}\left(\boldsymbol{x}, t ; \boldsymbol{x}^{\prime}, t^{\prime}\right) \nabla^{\prime} \times \boldsymbol{C}\left(\boldsymbol{x}^{\prime}, t^{\prime}\right) \mathrm{d} V^{\prime} \mathrm{d} t^{\prime}, \\
& \boldsymbol{C}_{3}(\boldsymbol{x}, t)=\frac{1}{4 \pi c} \frac{\partial}{\partial t} \int G_{d}\left(\boldsymbol{x}, t ; \boldsymbol{x}^{\prime}, t^{\prime}\right) \frac{\partial \boldsymbol{C}\left(\boldsymbol{x}^{\prime}, t^{\prime}\right)}{\partial t^{\prime}} \mathrm{d} V^{\prime} \mathrm{d} t^{\prime} .
\end{aligned}
$$

QED

A somewhat different derivation of this theorem has been given by Davis [13] and the relation of his results to the ones presented here is discussed in [14]. Heras recognized [12] that the direct application of this result to the time dependent vectors of the electromagnetic field is not easy, because the rotational must be specified together with the time derivative according to a theorem proved by Kapucik [15]. But it can be used to obtain Jefimenko's formulas, and many other things as we have already said. However it seems that a Helmholtz-Maxwell hyperbolic theorem, i.e. to make a decomposition whose different parts satisfy Maxwell's equations, is not available because even if we can identify rotational and solenoidal components, we cannot do the same for the time component in such a way that we obtain independent sets of differential equations for each component. Indeed, if we apply directly Maxwell equations we obtain Jefimenko's formulas after a time integration [15]. However we can combine Maxwell's equations and theorem 2 to obtain a useful representation of the fields. So we have

Theorem 2A (Helmholtz-Maxwell Hyperbolic Theorem):

Three $S^{k}, k \geq 1$, time dependent vector fields $\boldsymbol{E}, \boldsymbol{B}$ and $\boldsymbol{J}$ and a scalar time-dependent field $\rho$ that satisfy the following conditions:

1) A decomposition according to theorem 2.

2) They satisfy Maxwell equations

3) $\rho \in S^{k}, k>1$

admit the following representation:

$$
\left.\begin{array}{l}
\boldsymbol{E}(\boldsymbol{x}, t)=-c \nabla \int G_{d}\left(\boldsymbol{x}, t ; \boldsymbol{x}^{\prime}, t^{\prime}\right) \varrho\left(\boldsymbol{x}^{\prime}, t^{\prime}\right) \mathrm{d} V^{\prime} \mathrm{d} t^{\prime}+\frac{1}{c^{2}} \frac{\partial}{\partial t} \int G_{d}\left(\boldsymbol{x}, t ; \boldsymbol{x}^{\prime}, t^{\prime}\right) \boldsymbol{J}\left(\boldsymbol{x}^{\prime}, t^{\prime}\right) \mathrm{d} V^{\prime} \mathrm{d} t^{\prime} \\
\boldsymbol{B}(\boldsymbol{x}, t)=\frac{4 \pi}{c} \int G_{d}\left(\boldsymbol{x}, t ; \boldsymbol{x}^{\prime}, t^{\prime}\right) \nabla^{\prime} \times \boldsymbol{J}\left(\boldsymbol{x}^{\prime}, t^{\prime}\right) \mathrm{d} V^{\prime} \mathrm{d} t^{\prime}
\end{array}\right\}
$$

Proof: again the proof is straightforward, we use the theorem 2 to calculate the components of the electric and magnetic time dependent vectors, then we introduce Maxwell equations. The first component of the electric field in HMHT2A is obvious, but let us work the second and third components of decomposition (14): 


$$
\begin{aligned}
\boldsymbol{E}_{2}(\boldsymbol{x}, t) & =\frac{c}{4 \pi} \nabla \times \int G_{d}\left(\boldsymbol{x}, t ; \boldsymbol{x}^{\prime}, t^{\prime}\right) \nabla^{\prime} \times \boldsymbol{E}\left(\boldsymbol{x}^{\prime}, t^{\prime}\right) \mathrm{d} V^{\prime} \mathrm{d} t^{\prime} \\
& =\frac{c}{4 \pi} \int \nabla \times\left(G_{d}\left(\boldsymbol{x}, t ; \boldsymbol{x}^{\prime}, t^{\prime}\right) \nabla^{\prime} \times \boldsymbol{E}\left(\boldsymbol{x}^{\prime}, t^{\prime}\right)\right) \mathrm{d} V^{\prime} \mathrm{d} t^{\prime} \\
& =-\frac{1}{4 \pi} \int \nabla \times\left(G_{d}\left(\boldsymbol{x}, t ; \boldsymbol{x}^{\prime}, t^{\prime}\right) \frac{\partial \boldsymbol{B}\left(\boldsymbol{x}^{\prime}, t^{\prime}\right)}{\partial t^{\prime}}\right) \mathrm{d} V^{\prime} \mathrm{d} t^{\prime} \\
& =-\frac{1}{4 \pi} \int \nabla G_{d}\left(\boldsymbol{x}, t ; \boldsymbol{x}^{\prime}, t^{\prime}\right) \times \frac{\partial \boldsymbol{B}\left(\boldsymbol{x}^{\prime}, t^{\prime}\right)}{\partial t^{\prime}} \mathrm{d} V^{\prime} \mathrm{d} t^{\prime} \\
& =\frac{1}{4 \pi} \int \nabla^{\prime} G_{d}\left(\boldsymbol{x}, t ; \boldsymbol{x}^{\prime}, t^{\prime}\right) \times \frac{\partial \boldsymbol{B}\left(\boldsymbol{x}^{\prime}, t^{\prime}\right)}{\partial t^{\prime}} \mathrm{d} V^{\prime} \mathrm{d} t^{\prime} \\
& =-\frac{1}{4 \pi} \int G_{d}\left(\boldsymbol{x}, t ; \boldsymbol{x}^{\prime}, t^{\prime}\right) \frac{\partial}{\partial t^{\prime}} \nabla^{\prime} \times \mathbf{B}\left(\boldsymbol{x}^{\prime}, t^{\prime}\right) \mathrm{d} V^{\prime} \mathrm{d} t^{\prime} \\
& =-\frac{1}{4 \pi} \int \frac{\partial G_{d}\left(\boldsymbol{x}, t ; \boldsymbol{x}^{\prime}, t^{\prime}\right)}{\partial t} \nabla^{\prime} \times \boldsymbol{B}\left(\boldsymbol{x}^{\prime}, t^{\prime}\right) \mathrm{d} V^{\prime} \mathrm{d} t^{\prime} \\
& =-\frac{1}{4 \pi} \frac{\partial}{\partial t} \int G_{d}\left(\boldsymbol{x}, t ; \boldsymbol{x}^{\prime}, t^{\prime}\right) \nabla^{\prime} \times \boldsymbol{B}\left(\boldsymbol{x}^{\prime}, t^{\prime}\right) .
\end{aligned}
$$

As usual we have discarded surface terms and time integrations over the whole infinite time interval. The time component becomes:

$$
\boldsymbol{E}_{3}(\boldsymbol{x}, t)=\frac{1}{4 \pi c} \frac{\partial}{\partial t} \int G_{d}\left(\boldsymbol{x}, t ; \boldsymbol{x}^{\prime}, t^{\prime}\right) \frac{\partial \boldsymbol{E}\left(\boldsymbol{x}^{\prime}, t^{\prime}\right)}{\partial t^{\prime}} \mathrm{d} V^{\prime} \mathrm{d} t^{\prime}
$$

So combining with $\boldsymbol{E}_{2}(\boldsymbol{x}, t)$ and using Maxwell equations we get:

$$
\begin{aligned}
\boldsymbol{E}_{2}(\boldsymbol{x}, t)+\boldsymbol{E}_{3}(\boldsymbol{x}, t) & =\frac{1}{4 \pi} \frac{\partial}{\partial t} \int G_{d}\left(\boldsymbol{x}, t ; \boldsymbol{x}^{\prime}, t^{\prime}\right)\left(-\nabla^{\prime} \times \boldsymbol{B}\left(\boldsymbol{x}^{\prime}, t^{\prime}\right)+\frac{1}{c} \frac{\partial \boldsymbol{E}\left(\boldsymbol{x}^{\prime}, t^{\prime}\right)}{\partial t^{\prime}}\right) \mathrm{d} V^{\prime} \mathrm{d} t^{\prime} \\
& =\frac{1}{c^{2}} \frac{\partial}{\partial t} \int G_{d}\left(\boldsymbol{x}, t ; \boldsymbol{x}^{\prime}, t^{\prime}\right) \boldsymbol{J}\left(\boldsymbol{x}^{\prime}, t^{\prime}\right) \mathrm{d} V^{\prime} \mathrm{d} t^{\prime} .
\end{aligned}
$$

To obtain the representation for the magnetic field almost the same steps are followed, hence we may skip them. QED

The differences between HMHT and HMET are quite obvious, indeed, most of them lie in the instantaneous term, while others in the use of the solenoidal component of the current in HMET.

It is possible a formulation of Helmholtz theorem in Minkowski space-time using the electromagnetic field tensor $F^{\mu \sigma}$, a tensor that can be decomposed using some known tensor identities. This is the way followed by Kobe [14] using a decomposition theorem for the field tensor in such a way that Maxwell equations can be directly applied and a 4 dimensional solution obtained. For this reason one is led to believe that each Helmholtz decomposition is natural for a space-time geometry. Now we shall close this section with a "Helmholtz Parabolic Theorem".

\section{Theorem 3 (Helmholtz Parabolic Theorem)}

A $S^{k}, k \geq 1$, time dependent vector field defined over all of a Euclidean space-time may be uniquely represented as a sum of an irrotational, a solenoidal and a time part, up to an additive constant.

Proof: The proof is like the one for the hyperbolic theorem, except that the vector identity required is:

$$
\left(\Delta-D \frac{\partial}{\partial t}\right) \boldsymbol{C}=\nabla(\nabla \cdot \boldsymbol{C})-\nabla \times \nabla \times \boldsymbol{C}-D \frac{\partial \boldsymbol{C}}{\partial t} .
$$

Therefore we obtain the following formula:

$$
\boldsymbol{C}(\boldsymbol{x}, t)=-\frac{C}{4 \pi} \int G_{f}\left(\boldsymbol{x}, t ; \boldsymbol{x}^{\prime}, t^{\prime}\right)\left(\nabla^{\prime}\left(\nabla^{\prime} \cdot \boldsymbol{C}\left(\boldsymbol{x}^{\prime}, t^{\prime}\right)\right)-\nabla^{\prime} \times \nabla^{\prime} \times \boldsymbol{C}\left(\boldsymbol{x}^{\prime}, t^{\prime}\right)-D \frac{\partial \boldsymbol{C}\left(\boldsymbol{x}^{\prime}, t^{\prime}\right)}{\partial t^{\prime}}\right) \mathrm{d} V^{\prime} \mathrm{d} t^{\prime}
$$

So, we easily get: 


$$
C(x, t)=C_{p 1}(x, t)+C_{p 2}(x, t)+C_{p 3}(x, t),
$$

where

$$
\begin{aligned}
& C_{p 1}(x, t)=-\frac{c}{4 \pi} \nabla \int G_{f}\left(x, t ; x^{\prime}, t^{\prime}\right) \nabla^{\prime} \cdot \boldsymbol{C}\left(\boldsymbol{x}^{\prime}, t^{\prime}\right) \mathrm{d} V^{\prime} \mathrm{d} t^{\prime} \\
& C_{p 2}(x, t)=\frac{c}{4 \pi} \nabla \times \int G_{f}\left(x, t ; x^{\prime}, t^{\prime}\right) \nabla^{\prime} \times C\left(x^{\prime}, t^{\prime}\right) \mathrm{d} V^{\prime} \mathrm{d} t^{\prime} \\
& C_{p 3}(x, t)=\frac{D c}{4 \pi} \frac{\partial}{\partial t} \int G_{f}\left(x, t ; x^{\prime}, t^{\prime}\right) C\left(x^{\prime}, t^{\prime}\right) \mathrm{d} V^{\prime} \mathrm{d} t .
\end{aligned}
$$

QED

The time part is not like the time part for theorem 2.

\section{Theorem 3A (Helmholtz-Maxwell Parabolic Theorem)}

Three $S^{k}, k \geq 1$, time dependent vector fields $\boldsymbol{E}, \boldsymbol{B}$ and $\boldsymbol{J}$ and a scalar time-dependent field $\rho$ that satisfy the following conditions:

1) A decomposition according to theorem 3.

2) They satisfy Maxwell equations

3) $\rho \in S^{k}, k>1$

admit the following representation:

$$
\left.\begin{array}{l}
\boldsymbol{E}(\boldsymbol{x}, t)=-\frac{c}{4 \pi} \nabla \int G_{f}\left(\boldsymbol{x}, t ; \boldsymbol{x}^{\prime}, t^{\prime}\right) \varrho\left(\boldsymbol{x}^{\prime}, t^{\prime}\right) \mathrm{d} V^{\prime} \mathrm{d} t^{\prime}-\frac{1}{c} \frac{\partial}{\partial t} \int G_{f}\left(\boldsymbol{x}, t ; \boldsymbol{x}^{\prime}, t^{\prime}\right) \boldsymbol{J}_{T}\left(\boldsymbol{x}^{\prime}, t^{\prime}\right) \mathrm{d} V^{\prime} \mathrm{d} t^{\prime} \\
\boldsymbol{B}(\boldsymbol{x}, t)=\frac{4 \pi}{c} \int G_{d}\left(\boldsymbol{x}, t ; \boldsymbol{x}^{\prime}, t^{\prime}\right) \nabla^{\prime} \times \boldsymbol{J}_{T}\left(\boldsymbol{x}^{\prime}, t^{\prime}\right) \mathrm{d} V^{\prime} \mathrm{d} t
\end{array}\right\}
$$

where

$$
\boldsymbol{J}_{T}=\boldsymbol{J}+\frac{1}{4 \pi} \frac{\partial \boldsymbol{E}}{\partial t}-\frac{D c^{2}}{4 \pi} \boldsymbol{E}\left(\boldsymbol{x}^{\prime}, t^{\prime}\right) .
$$

This current we may call "total current”, because it includes the usual Maxwell displacement current, the current density and something that, when conductors are considered, may be called Ohm's current because of Ohm's law.

Proof: we apply theorem 3 to the electromagnetic field. We obtain:

$$
\boldsymbol{E}(\boldsymbol{x}, t)=\boldsymbol{E}_{p 1}(\boldsymbol{x}, t)+\boldsymbol{E}_{p 2}(\boldsymbol{x}, t)+\boldsymbol{E}_{p 3}(\boldsymbol{x}, t) .
$$

Using Maxwell's Equation (2) we get:

$$
\boldsymbol{E}_{p 1}(\boldsymbol{x}, t)=-\frac{c}{4 \pi} \nabla \int G_{f}\left(\boldsymbol{x}, t ; \boldsymbol{x}^{\prime}, t^{\prime}\right) \varrho\left(\boldsymbol{x}^{\prime}, t^{\prime}\right) \mathrm{d} V^{\prime} \mathrm{d} t^{\prime} .
$$

But we can write down:

$$
\boldsymbol{E}_{p 2}(\boldsymbol{x}, t)=-\frac{1}{4 \pi} \frac{\partial}{\partial t} \int G_{f}\left(\boldsymbol{x}, t ; \boldsymbol{x}^{\prime}, t^{\prime}\right) \nabla^{\prime} \times \boldsymbol{B}\left(\boldsymbol{x}^{\prime}, t^{\prime}\right) \mathrm{d} V^{\prime} \mathrm{d} t^{\prime} .
$$

So we have a time component:

$$
\boldsymbol{E}_{p 2}(\boldsymbol{x}, t)+\boldsymbol{E}_{p 3}(\boldsymbol{x}, t)=\frac{1}{4 \pi} \frac{\partial}{\partial t} \int G_{f}\left(\boldsymbol{x}, t ; \boldsymbol{x}^{\prime}, t^{\prime}\right)\left(-\nabla^{\prime} \times \boldsymbol{B}\left(\boldsymbol{x}^{\prime}, t^{\prime}\right)+D c \boldsymbol{E}\left(\boldsymbol{x}^{\prime}, t^{\prime}\right)\right) \mathrm{d} V^{\prime} \mathrm{d} t^{\prime} .
$$

Next we apply Maxwell equations and we obtain the desired representation for the electric field. We skip the calculations for the magnetic field because they are easy. QED

Example: We can use TP to solve the electromagnetic problem in at least two situations: the electric field in a conducting media or in a superconducting one, because in both cases we have relations among the fields and the currents. For superconductors we must take as zero, in Maxwell equations, the displacement current, and suppose the validity of London's equations which we shall take as: 


$$
\begin{gathered}
\nabla \times \boldsymbol{B}=\frac{4 \pi}{c} \boldsymbol{J}_{u} \\
\frac{4 \pi}{c} \nabla \times \boldsymbol{J}_{u}=-\frac{1}{l} \boldsymbol{B} \\
\frac{4 \pi}{c} \frac{\partial \boldsymbol{J}_{u}}{\partial t}=\frac{1}{l} \boldsymbol{E} .
\end{gathered}
$$

As usual $l$ is London's penetration depth, while $\boldsymbol{J}_{u}$ is the superconducting current.

$$
\boldsymbol{E}_{p 2}^{s}(\boldsymbol{x}, t)+\boldsymbol{E}_{p 3}^{s}(\boldsymbol{x}, t)=\frac{1}{c} \frac{\partial}{\partial t} \int G_{f}\left(\boldsymbol{x}, t ; \boldsymbol{x}^{\prime}, t^{\prime}\right)\left(\frac{1}{c} \boldsymbol{J}_{u}\left(\boldsymbol{x}^{\prime}, t^{\prime}\right)+l D \frac{\partial \boldsymbol{J}_{u}\left(\boldsymbol{x}^{\prime}, t^{\prime}\right)}{\partial t^{\prime}}\right) \mathrm{d} V^{\prime} \mathrm{d} t^{\prime} .
$$

For the case of conductors we use Ohm's law and Maxwell equations for conducting media, but the result is formally analogous to (16) so we skip it.

\section{Helmholtz Theorems and Gauges Transformations for the Electromagnetic Potentials}

In the previous section we have introduced several decompositions that deserve the name of Helmholtz, and we have discussed the solutions that each one of them allows us of the Maxwell's equations. Such a combination of Maxwell's equations and Helmholtz decompositions we have called "Helmholtz-Maxwell theorems" and we have used them to obtain a particular representation of the fields whose usefulness will become clear in this section. We have supposed that all the solutions obtained are different solutions that arise out of different sets of premises - e.g. the elliptic Helmholtz representation arise form an elliptic equation, while the hyperbolic Helmholtz representation comes from solving hyperbolic equations. There is no reason to suppose that all these solutions are one and the same because we have been working with the field strengths, and different suppositions must give us different, probably unrelated, fields. However, we know that solutions can be related if we make use of Helmholtz decompositions, being the reduction of the retarded solution to instantaneous one, an instance of this process. That is, the Equation (1), even if formally hyperbolic, are just differential consequences of Maxwell's equations, so, when combined with a Helmholtz decomposition like that of theorem B become elliptic equations with instantaneous solutions. The HMET is the formalization of this procedure and makes it clear what is going on. Clearly, if we combine the Maxwell's equations with a different Helmholtz decomposition, like that of theorem 2 we shall get retarded solutions only. Now we are going to discuss the process using potentials instead of field strengths because, as we shall see, with them we can identify a Helmholtz decomposition with a specific gauge transformation.

The differential equations that satisfy the potentials are invariant under the action of an infinite dimensional group whose orbits are labeled by continuous functions, with the consequence that any solution includes arbitrary functions, making the system formally indeterministic and the solutions quite arbitrary (a neat discussion of gauge systems in [16]). Hence the potentials $\langle\boldsymbol{A}, \varphi\rangle$ were considered, for a long time, quite a mathematical delusion useful to solve Maxwell's equations, being the physically relevant quantities the gauge invariant ones, like the field strengths. The gauge invariant equations for the potentials, in Gibbs' three-dimensional notation, are:

$$
\begin{gathered}
\nabla \times \nabla \times \boldsymbol{A}=-\frac{1}{c} \frac{\partial}{\partial t} \nabla \varphi-\frac{1}{c^{2}} \frac{\partial^{2} \boldsymbol{A}}{\partial t^{2}}+\frac{4 \pi}{c} \boldsymbol{J} \\
\Delta \varphi+\frac{1}{c} \frac{\partial}{\partial t} \nabla \cdot \boldsymbol{A}=-4 \pi \varrho .
\end{gathered}
$$

While the equations for the field strengths are:

$$
\begin{gathered}
\boldsymbol{E}=-\nabla \varphi-\frac{1}{c} \frac{\partial \boldsymbol{A}}{\partial t} \\
\boldsymbol{B}=\nabla \times \boldsymbol{A} .
\end{gathered}
$$


Quite directly these are not easy to solve equations, and for that reason a procedure to obtain its solution was designed, consisting in the imposition of conditions between the potentials and its derivatives-known as "gauges". In this way the equations for the potentials can be solved. This procedure is justified supposing that we can always choose a gauge transformation from the original solutions of the differential equations $\langle A, \varphi\rangle$ to those in a particular gauge $\left\langle\boldsymbol{A}_{g}, \varphi_{g}\right\rangle$. In this way all gauges are related by means of gauge transformations of the form:

$$
\begin{gathered}
\boldsymbol{A}=\boldsymbol{A}_{g}+\nabla \gamma \\
\varphi=\varphi_{g}-\frac{1}{c} \frac{\partial \gamma}{\partial t},
\end{gathered}
$$

where $\gamma: \mathbf{R}^{4} \rightarrow \mathbf{R}^{1}$ is an arbitrary function labeling the group orbit and the origin of the infinite dimensionality of the symmetry group. See reference [16] for examples of these gauge functions. From the previous explanations it should be clear that, as a result of the definitions, the following theorem should be obvious: The gauge transformations cannot affect the gauge group invariants, hence all the solutions of Maxwell equations obtained in any gauge are the same modulo a gauge function. In particular, the time dependent vectors representing the electromagnetic field should be the same no matter the gauge of the potentials. Doubts on the validity of the theorem arise because in the Coulomb gauge the scalar potential is instantaneous, while in the Lorenz gauge is a retarded quantity. So, as Jackson wrote: It seems necessary from time to time to show that the electric and magnetic fields are independent of the choice of gauge for the potentials" [17], and Heras agreed [18]. Clearly if we suppose that the potentials are physically relevant quantities, as the Aharonov-Bohm effect seems to suggest, gauge invariance, as a criterion of physical relevance would be sufficient but probably not a necessary one. And if the potentials are physically relevant quantities it can be supposed that some of them, or all, satisfy the same causality conditions than the fields (an excellent discussion is [19]). Our purpose in this section is to prove that a gauge transformation of the potentials is equivalent to a Helmholtz theorem, at least for the Helmholtz decompositions we have discussed. If we have seen carefully the Helmholtz-Maxwell theorems we discover that the structure of the representation is quite the same as the one given by Equations ((19), (20)), with the following definitions:

$$
\begin{aligned}
& \boldsymbol{A}_{C}(\boldsymbol{x}, t)=-\frac{4 \pi}{c} \int G_{d}\left(\boldsymbol{x}, t ; \boldsymbol{x}^{\prime}, t^{\prime}\right) \boldsymbol{J}_{S}\left(\boldsymbol{x}^{\prime}, t^{\prime}\right) \mathrm{d} V^{\prime} \mathrm{d} t^{\prime} \\
& \boldsymbol{A}_{L}(\boldsymbol{x}, t)=-\frac{4 \pi}{c} \int G_{d}\left(\boldsymbol{x}, t ; \boldsymbol{x}^{\prime}, t^{\prime}\right) \boldsymbol{J}\left(\boldsymbol{x}^{\prime}, t^{\prime}\right) \mathrm{d} V^{\prime} \mathrm{d} t^{\prime} \\
& \boldsymbol{A}_{P}(\boldsymbol{x}, t)=-\frac{4 \pi}{c} \int G_{f}\left(\boldsymbol{x}, t ; \boldsymbol{x}^{\prime}, t^{\prime}\right) \boldsymbol{J}_{T}\left(\boldsymbol{x}^{\prime}, t^{\prime}\right) \mathrm{d} V^{\prime} \mathrm{d} t^{\prime} .
\end{aligned}
$$

If these solutions come from a gauge all of them are related by means of a gauge transformation. We can see that if we define:

$$
\begin{aligned}
& \varphi_{C}(x, t)=\int G_{l}\left(x, t ; x^{\prime}, t^{\prime}\right) \varrho\left(x^{\prime}, t^{\prime}\right) \mathrm{d} V^{\prime} \mathrm{d} t^{\prime} \\
& \varphi_{L}(x, t)=\int G_{d}\left(x, t ; x^{\prime}, t^{\prime}\right) \varrho\left(x^{\prime}, t^{\prime}\right) \mathrm{d} V^{\prime} \mathrm{d} t^{\prime} \\
& \varphi_{L}(x, t)=\frac{C}{4 \pi} \int G_{f}\left(x, t ; x^{\prime}, t^{\prime}\right) \varrho\left(x^{\prime}, t^{\prime}\right) \mathrm{d} V^{\prime} \mathrm{d} t^{\prime} .
\end{aligned}
$$

We get:

$$
\begin{aligned}
& \nabla \cdot \boldsymbol{A}_{C}(\boldsymbol{x}, t)=0 \\
& \nabla \cdot \boldsymbol{A}_{L}(\boldsymbol{x}, t)=-\frac{1}{c} \frac{\partial \varphi_{L}(\boldsymbol{x}, t)}{\partial t} \\
& \nabla \cdot \boldsymbol{A}_{P}(\boldsymbol{x}, t)=-\frac{4 \pi}{C} D \varphi_{P}(\boldsymbol{x}, t) .
\end{aligned}
$$


These equations show that the functions (21)-(26) we have introduced satisfy different sets of conditions that we can identify with typical gauge conditions. Because our solutions were obtained using Helmholtz decompositions is clear that a gauge can be correlated to a Helmholtz decomposition. Of course, Heras is right when he thinks that we prescribe the Green's function in each different decomposition, but we do something else, we prescribe the gauge condition as well. In essence, we are prescribing the divergence of the vector potential. Now it is easy to see by inspection the sets of equations that the functions (21)-(24), (22)-(25), (23)-(26) satisfy; or we can obtain them systematically by using the gauges conditions (27)-(29) in Equations (17)-(18). For the Coulomb gauge (27) and the Lorenz gauge (28) these equations are well-known, but for the "parabolic gauge" (29) it is probably worth writing them down. These equations are:

$$
\begin{aligned}
& \left(\Delta-D \frac{\partial}{\partial t}\right) \boldsymbol{A}_{P}=-\frac{4 \pi}{c} D \boldsymbol{J}_{T} \\
& \left(\Delta-\frac{4 \pi}{c^{2}} D \frac{\partial}{\partial t}\right) \varphi_{P}=-4 \pi \varrho .
\end{aligned}
$$

Here $\boldsymbol{J}_{T}$ is the total current (15). In the Coulomb gauge the instantaneous solution is big trouble, in the parabolic gauge we have an irreversible behavior of the solutions because the equations are not invariant under time reversal. All solutions in the Lorenz gauge are invariant under time reversal and retarded, so the described phenomena are reversible in the thermodynamic sense, so, there is no natural room for irreversibility, however this important feature of the world — the irreversibility—is present in the parabolic gauge, and is an "element of reality" that is lost when we use a gauge transformation to change the gauge.

Now it is clear that each Helmholtz decomposition is related to a different gauge, so we need to discover what the precise relationship between a gauge and a Helmholtz decomposition is. For short, the sort of Helmholtz decomposition achieved using theorem B of section III, above, we shall call "elliptic decomposition".

Let us start with the following theorem:

Theorem 4: Any gauge transformation to the Coulomb gauge of a $S^{k}$ time dependent vector potential field $\boldsymbol{A}$ in any gauge or no gauge at all, is an elliptic Helmholtz decomposition if the following conditions are satisfied:

1) $\gamma_{C} \in S^{k}, k>1$

2) $\boldsymbol{A}_{C}$, the potential in the Coulomb gauge, is $S^{k}$

where $\gamma_{C}$ is the gauge.

Proof: The gauge transformation to the Coulomb gauge is:

$$
\boldsymbol{A}=\boldsymbol{A}_{C}+\nabla \gamma_{C} .
$$

What we must prove is that each of the components in (30) is exactly given by (HT). This we can do in the following way. We take the divergence of (30) to get:

$$
\nabla \cdot \boldsymbol{A}=\Delta \gamma_{C}
$$

So we get easily by solving the boundary value problem under assumption (1) in the theorem:

$$
\gamma_{C}(\boldsymbol{x}, t)=\int G_{l}\left(\boldsymbol{x}, t ; \boldsymbol{x}^{\prime}, \boldsymbol{t}^{\prime}\right) \nabla^{\prime} \cdot \boldsymbol{A}\left(\boldsymbol{x}^{\prime}, t^{\prime}\right) \mathrm{d} V^{\prime} \mathrm{d} t^{\prime} .
$$

Which is what we wanted. Now we take the double rotational of Equation (30) and applying the gauge condition we get:

$$
\nabla \times \nabla \times \boldsymbol{A}=\nabla \times \nabla \times \boldsymbol{A}_{C}=-\Delta \boldsymbol{A}_{C} .
$$

Therefore, again solving the boundary value problem:

$$
\boldsymbol{A}_{C}(\boldsymbol{x}, t)=\int G_{l}\left(\boldsymbol{x}, t ; \boldsymbol{x}^{\prime}, t^{\prime}\right) \nabla^{\prime} \times \nabla^{\prime} \times \boldsymbol{A}\left(\boldsymbol{x}^{\prime}, \boldsymbol{t}^{\prime}\right) \mathrm{d} V^{\prime} \mathrm{d} t^{\prime} .
$$

Using well-known vector identities and properties of the Green's function G2, we finally obtain:

$$
\boldsymbol{A}_{C}(\boldsymbol{x}, t)=\nabla \times \int G_{l}\left(\boldsymbol{x}, t ; \boldsymbol{x}^{\prime}, t^{\prime}\right) \nabla^{\prime} \times \boldsymbol{A}\left(\boldsymbol{x}^{\prime}, t^{\prime}\right) \mathrm{d} V^{\prime} \mathrm{d} t^{\prime} .
$$

So a gauge transformation from an arbitrary vector potential $\boldsymbol{A}$ to a vector potential in the Coulomb gauge $\boldsymbol{A}_{C}$ is an elliptic Helmholtz decomposition of the vector $\boldsymbol{A}$. This proves the theorem. QED 
Theorem 5: An elliptic Helmholtz decomposition of the time dependent $S^{k}$ vector potential and the $S^{k}$ current density in Euclidean 3-space that satisfy Maxwell equations is, locally, a gauge transformation to the Coulomb gauge, that induces the corresponding gauge transformation of the $S^{k}$ scalar potential.

Comment I: Hence an elliptic Helmholtz decomposition is a sufficient condition to transform any potential that satisfies Maxwell equations to the Coulomb gauge, and we obtain the Helmholtz-Maxwell representation of theorem (1) above. In this and subsequent theorems "local" means that the transformation is valid for path connected components of the space-time. Hence it is globally defined on space-times with closed paths homotopic to zero only.

Proof: We must start from an elliptic Helmholtz decomposition of the vector potential and, with the help of Maxwell Equations ((17), (18)), to prove that each component satisfy the differential equations of the Coulomb gauge. So we start with the elliptic Helmholtz decomposition of an arbitrary vector potential in the form: $\boldsymbol{A}=\boldsymbol{A}_{s}+\boldsymbol{A}_{i}$, such that: $\nabla \cdot \boldsymbol{A}_{s}=0, \nabla \times \boldsymbol{A}_{i}=0$. Locally we can express the irrotational component in the form: $A_{i}=-\nabla G_{i}$ a representation that is going to be very useful. Now we put the decomposition in the Equations ((17), (18)) to get, after identifying irrotational and solenoidal components-including an elliptic Helmholtz decomposition for the current density—-the following equations:

$$
\begin{gathered}
\left(\Delta-\frac{1}{c^{2}} \frac{\partial^{2}}{\partial t^{2}}\right) \boldsymbol{A}_{s}(\boldsymbol{x}, t)=-\frac{4 \pi}{c} \boldsymbol{J}_{s} \\
\Delta \Phi=-4 \pi \varrho \\
\frac{1}{c} \frac{\partial}{\partial t} \nabla \Phi=\frac{4 \pi}{c} \boldsymbol{J}_{i},
\end{gathered}
$$

where $\Phi=\varphi-\frac{1}{c} \frac{\partial G_{i}}{\partial t}$. These are the equations of the Coulomb gauge, hence $\left\langle\boldsymbol{A}_{s}, \Phi\right\rangle$ are the potentials in the Coulomb gauge. QED

In this way a gauge transformation to the Coulomb gauge is an elliptic Helmholtz decomposition, while an elliptic Helmholtz decomposition of the vector potential becomes a Helmholtz-Maxwell decomposition, which is a transformation to the Coulomb gauge.

Comment II: theorem (5) is not as trivial as theorem (4), and some ambiguities appeared around its interpretation. A careful derivation of the Equations ((31)-(33)) appeared under a different interpretation in [20], but in [21] it was proved that the equations are really the Coulomb gauge equations. In [20] it was also proved that the potentials $\left\langle\boldsymbol{A}_{s}, \Phi\right\rangle$ are gauge invariant quantities, so the Coulomb gauge is enough to fix the arbitrary degrees of freedom coming from gauge invariance of Equations ((17), (18)). Indeed, this proves that gauge invariance is probably a necessary criteria of reality, while it is not sufficient. Otherwise we should accept that the potentials in the Coulomb gauge are physically relevant quantities acting at a distance. So we have a conceptually unclear situation. If we suppose that, as the Aharonov-Bohm effect seems to suggest, the potentials are physically relevant quantities even if they are not gauge invariant, hence gauge invariance is not a sufficient criteria of reality. But, if on the other hand, we accept that the potentials in the Coulomb gauge are not physically relevant quantities-because they are instantaneous - even if they are gauge invariant, then gauge invariance is not a sufficient criteria of physical relevance. In the end, we conclude that gauge invariance is not relevant to determine physical reality of mathematical representations. Stewart in [22] applied the elliptic Helmholtz decomposition to the time dependent vector potential. Next, to obtain an analytic representation, he supposed that the vector potential was defined in the Coulomb gauge and his effort finalized proving that his representation satisfied Maxwell equations. With the help of theorem 4 we can see that if we suppose that the time dependent vector potential is already in the Coulomb gauge, no transformation is required, so the gauge function vanishes. Hence, we just have

$$
\boldsymbol{A}_{C}(\boldsymbol{x}, t)=\nabla \times \int G_{l}\left(\boldsymbol{x}, t ; \boldsymbol{x}^{\prime}, t^{\prime}\right) \boldsymbol{B}\left(\boldsymbol{x}^{\prime}, t^{\prime}\right) \mathrm{d} V^{\prime} \mathrm{d} t^{\prime} .
$$

Stewart showed that this representation of the time dependent vector potential satisfies Maxwell equations and even more, that it is equivalent to retarded representation obtained by Jackson [17]. However, its very easy to prove that (34) is in fact the potential (21) coming from the Helmholtz-Maxwell elliptic theorem. We have the following:

Corollary to theorem 5: the instantaneous Stewart representation (34) for the vector potential is equivalent to 
the retarded representation (21).

Proof: Using standard vector identities and Green's functions properties we can write down

$$
\begin{aligned}
\boldsymbol{A}_{C}(\boldsymbol{x}, t) & =-\frac{4 \pi}{c} \int G_{d}\left(\boldsymbol{x}, t ; \boldsymbol{x}^{\prime}, t^{\prime}\right) \boldsymbol{J}_{s}\left(\boldsymbol{x}^{\prime}, t^{\prime}\right) \mathrm{d} V^{\prime} \mathrm{d} t^{\prime} \\
& =\int G_{d}\left(\boldsymbol{x}, t ; \boldsymbol{x}^{\prime}, t^{\prime}\right)\left(\Delta^{\prime}-\frac{1}{c^{2}} \frac{\partial^{2}}{\partial t^{\prime 2}}\right) \boldsymbol{A}_{s}(\boldsymbol{x}, t) \mathrm{d} V^{\prime} \mathrm{d} t^{\prime} \\
& =\int\left(\Delta^{\prime}-\frac{1}{c^{2}} \frac{\partial^{2}}{\partial t^{\prime 2}}\right) G_{d}\left(\boldsymbol{x}, t ; \boldsymbol{x}^{\prime}, t^{\prime}\right) \boldsymbol{A}_{s}(\boldsymbol{x}, t) \mathrm{d} V^{\prime} \mathrm{d} t^{\prime} \\
& =-\frac{4 \pi}{c} \int \delta\left(\boldsymbol{x}-\boldsymbol{x}^{\prime}\right) \delta\left(t-t^{\prime}\right) \boldsymbol{A}_{s}(\boldsymbol{x}, \boldsymbol{t}) \mathrm{d} V^{\prime} \mathrm{d} t^{\prime} \\
& =\int \Delta^{\prime} G_{l}\left(\boldsymbol{x}, t ; \boldsymbol{x}^{\prime}, t^{\prime}\right) \boldsymbol{A}_{s}(\boldsymbol{x}, t) \mathrm{d} V^{\prime} \mathrm{d} t^{\prime} \\
& =\int G_{l}\left(\boldsymbol{x}, t ; \boldsymbol{x}^{\prime}, t^{\prime}\right) \Delta^{\prime} \boldsymbol{A}_{s}(\boldsymbol{x}, t) \mathrm{d} V^{\prime} \mathrm{d} t^{\prime} \\
& =\int G_{l}\left(\boldsymbol{x}, t ; \boldsymbol{x}^{\prime}, t^{\prime}\right) \nabla^{\prime} \times \nabla^{\prime} \times \boldsymbol{A}_{s}(\boldsymbol{x}, t) \mathrm{d} V^{\prime} \mathrm{d} t^{\prime} \\
& =\Delta \times \int G_{l}\left(\boldsymbol{x}, t ; \boldsymbol{x}^{\prime}, t^{\prime}\right) \nabla^{\prime} \times \boldsymbol{A}_{s}(\boldsymbol{x}, t) \mathrm{d} V^{\prime} \mathrm{d} t^{\prime} .
\end{aligned}
$$

Because $\boldsymbol{B}=\nabla \times \boldsymbol{A}_{s}$ the proof is complete. QED

So we have a completely instantaneous representation of the Coulomb gauge of the form:

$$
\left.\begin{array}{l}
\Delta \varphi_{C}=-4 \pi \varrho \\
\Delta \boldsymbol{A}_{C}=\frac{1}{c} \frac{\partial \boldsymbol{E}}{\partial t}+\frac{4 \pi}{c} \boldsymbol{J}
\end{array}\right\}
$$

A moment of reflection shows us that this representation is almost obvious in the Coulomb gauge.

Let us remark the role of the elliptic Helmholtz representation, which is one of Stewart premises. If we use a hyperbolic or parabolic Helmholtz decomposition, things are different because more terms arise. So, in the Coulomb gauge it is possible to write down a completely instantaneous representation of the solution without the use of gauge transformations, that is, we can use two elliptic Poisson like equations as it is done in equations (S). But now the question arises if a completely retarded representation is possible not using gauge transformations, because if we use them, such a retarded representation of the Coulomb gauge turns out to be the Lorenz gauge. We believe that this is not possible.

Now let us prove the analogue of theorems 4 and 5 but for the Lorenz gauge. It is natural the introduction of a Minkowski space-time $M=\left\langle\eta_{\mu \sigma}, \mathbf{R}^{4}\right\rangle$. So we have:

Theorem 6: Any gauge transformation to the Lorenz gauge of a $S^{k} 4$ vector potential field in any gauge or no gauge at all, is an hyperbolic Helmholtz decomposition if the following conditions are satisfied:

1) $\gamma_{L} \in S^{k}, k>1$

2) $A_{\mu}^{L}$ the potential in the Lorenz gauge is $S^{k}$ where $\gamma_{L}$ is the gauge.

Proof:

$$
\begin{gathered}
A_{\mu}=A_{\mu}^{L}+\partial_{\mu} \gamma_{L} \\
\partial^{\mu} A_{\mu}=\partial^{\mu} \partial_{\mu} \gamma_{L} \\
\gamma_{L}(x)=\int G_{d}\left(x, x^{\prime}\right) \partial^{\prime \mu} A_{\mu}\left(x^{\prime}\right) \mathrm{d} x^{\prime},
\end{gathered}
$$

where $\mathrm{d} x^{\prime}$ is shorthand for the 4-volume element.

Next we use the 4-curl to obtain:

$$
\partial^{\mu} A_{\sigma}-\partial^{\sigma} A_{\mu}=\partial^{\mu} A_{\sigma}^{L}-\partial^{\sigma} A_{\mu}^{L} \rightarrow F_{\mu \sigma}=F_{\mu \sigma}^{L} .
$$

Which is just the gauge invariance of the electromagnetic field tensor. But we have the following identity: 


$$
\partial_{\mu} F_{\mu \sigma}=\partial_{\mu} \partial^{\mu} A_{\sigma}-\partial_{\mu} \partial^{\sigma} A_{\mu}=\partial_{\mu} \partial^{\mu} A_{\sigma}-\partial^{\sigma} \partial_{\mu} A_{\mu}
$$

Because we have: $\partial_{\mu} A_{\mu}^{L}=0$ we get:

$$
\partial_{\mu} F_{\mu \sigma}=\partial_{\mu} \partial^{\mu} A_{\sigma}^{L} .
$$

So:

$$
A_{\sigma}^{L}(x)=\int G_{d}\left(x, x^{\prime}\right) \partial_{\mu} F_{\mu \sigma}\left(x^{\prime}\right) \mathrm{d} x^{\prime} .
$$

Hence we get the components of the hyperbolic Helmholtz decomposition in Minkowski space-time, equivalent, as proved by Woodside [3] to the already discussed representation of theorem 2. QED

Theorem 7: A hyperbolic Helmholtz decomposition of a $S^{k}$ 4-vector potential $A^{\mu}$ and the $S^{k}$ 4-current $j^{\mu}$ that satisfies Maxwell equations in Minkowski 4-space is a gauge transformation to the Lorenz gauge.

Proof: We make the decomposition:

$$
A_{\mu}=A_{\mu}^{s}+A_{\mu}^{i}
$$

Such that:

$$
\partial^{\mu} A_{\sigma}^{i}-\partial^{\sigma} A_{\mu}^{i}=0, \partial_{\mu} A_{\mu}^{s}=0 .
$$

Now, Maxwell equations in Minkowski space-time are:

$$
\begin{gathered}
F_{\mu \sigma}=\partial^{\mu} A_{\sigma}-\partial^{\sigma} A_{\mu} \\
\partial_{\sigma} F_{\mu \sigma}=-\frac{4 \pi}{c} j_{\mu} \rightarrow \partial_{\sigma} \partial^{\mu} A_{\sigma}-\partial_{\sigma} \partial^{\sigma} A_{\mu}=-\frac{4 \pi}{c} j_{\mu} .
\end{gathered}
$$

Next we insert decomposition (39) in (42) to get:

$$
\partial_{\sigma} \partial^{\mu}\left(A_{\sigma}^{s}+A_{\sigma}^{i}\right)-\partial_{\sigma} \partial^{\sigma}\left(A_{\mu}^{s}+A_{\mu}^{i}\right)=-\frac{4 \pi}{c}\left(j_{\mu}^{s}+j_{\mu}^{i}\right) .
$$

Identifying rotational and solenoidal components, we finally write down:

$$
\begin{aligned}
& \partial_{\sigma} \partial^{\sigma} A_{\mu}^{s}=\frac{4 \pi}{c} j_{\mu}^{s}, \partial_{\sigma} A_{\sigma}^{s}=0 \\
& \partial_{\sigma} \partial^{\mu} A_{\sigma}^{i}-\partial_{\sigma} \partial^{\sigma} A_{\mu}^{i}=-\frac{4 \pi}{c} j_{\mu}^{i} .
\end{aligned}
$$

But:

$$
\partial^{\mu} A_{\sigma}^{i}=\partial^{\sigma} A_{\mu}^{i}
$$

So (45) becomes:

$$
j_{\mu}^{i}=0 .
$$

It is easy to see that (44) are the Lorenz gauge equations. QED

Indeed the use of a Minkowski space-time is very abstract and we cannot see the equivalence with theorem 2-the Hyperbolic Helmholtz decomposition. To see it, we start from the gauge transformation:

$$
\begin{gathered}
\boldsymbol{A}=\boldsymbol{A}_{L}+\nabla \gamma_{L} \\
\varphi=\varphi_{L}-\frac{1}{c} \frac{\partial \gamma_{L}}{\partial t} .
\end{gathered}
$$

To the Lorenz gauge. The pair (46)-(47) is equivalent to (35) but we are using now Gibbs' vector notation for 3 -space. Therefore we get from (46) and the Lorenz gauge's condition:

$$
\nabla \times \nabla \times \boldsymbol{A}=-\nabla\left(\frac{1}{c} \frac{\partial \varphi_{L}}{\partial t}\right)-\Delta \boldsymbol{A}_{L}
$$


But $\boldsymbol{E}=-\nabla \varphi_{L}-\frac{1}{c} \frac{\partial \boldsymbol{A}_{L}}{\partial t}$ so we obtain:

$$
\nabla \times \nabla \times \boldsymbol{A}=\frac{1}{c} \frac{\partial \boldsymbol{E}}{\partial t}-\left(\Delta-\frac{1}{c^{2}} \frac{\partial^{2}}{\partial t^{2}}\right) \boldsymbol{A}_{L} .
$$

And on the other hand we have:

$$
\nabla \cdot \boldsymbol{A}=-\frac{1}{c} \frac{\partial \varphi_{L}}{\partial t}+\Delta \gamma_{L}
$$

Inserting (47) it is:

$$
\nabla \cdot \boldsymbol{A}=-\frac{1}{c} \frac{\partial \varphi}{\partial t}=-\left(\Delta-\frac{1}{c^{2}} \frac{\partial^{2}}{\partial t^{2}}\right) \gamma_{L}
$$

From Equations ((48), (49)) we obtain the following decomposition of an arbitrary vector potential $A$ :

$$
\begin{aligned}
\boldsymbol{A}(\boldsymbol{x}, t)= & \nabla \int G_{d}\left(\boldsymbol{x}, t ; \boldsymbol{x}^{\prime}, t^{\prime}\right)\left(\nabla^{\prime} \cdot \boldsymbol{A}\left(\boldsymbol{x}^{\prime}, t^{\prime}\right)+\frac{1}{c} \frac{\partial \varphi\left(\boldsymbol{x}^{\prime}, t^{\prime}\right)}{\partial t^{\prime}}\right) \mathrm{d} V^{\prime} \mathrm{d} t^{\prime} \\
& -\nabla \times \int G_{d}\left(\boldsymbol{x}, t ; \boldsymbol{x}^{\prime}, t^{\prime}\right) \nabla^{\prime} \times \boldsymbol{A}\left(\boldsymbol{x}^{\prime}, t^{\prime}\right) \mathrm{d} V^{\prime} \mathrm{d} t^{\prime} \\
& +\frac{1}{c} \int G_{d}\left(\boldsymbol{x}, t ; \boldsymbol{x}^{\prime}, t^{\prime}\right) \frac{\partial \mathbf{E}\left(\boldsymbol{x}^{\prime}, t^{\prime}\right)}{\partial t^{\prime}} \mathrm{d} V^{\prime} \mathrm{d} t^{\prime} .
\end{aligned}
$$

We can see that if we use theorem 2-the Hyperbolic Helmholtz theorem-to obtain a decomposition of the vector potential we obtain quite the same as Equation (50) except for the terms involving the arbitrary scalar potential $\varphi$. To be clear, we get:

$$
\begin{aligned}
\boldsymbol{A}(\boldsymbol{x}, t)= & -\frac{c}{4 \pi} \nabla \int G_{d}\left(\boldsymbol{x}, t ; \boldsymbol{x}^{\prime}, t^{\prime}\right) \nabla \cdot \boldsymbol{A}\left(\boldsymbol{x}^{\prime}, t^{\prime}\right) \mathrm{d} V^{\prime} \mathrm{d} t^{\prime} \\
& +\frac{c}{4 \pi} \nabla \times \int G_{d}\left(\boldsymbol{x}, t ; \boldsymbol{x}^{\prime}, t^{\prime}\right) \boldsymbol{A}\left(\boldsymbol{x}^{\prime}, t^{\prime}\right) \mathrm{d} V^{\prime} \mathrm{d} t^{\prime} \\
& +\frac{1}{4 \pi c} \frac{\partial}{\partial t} \int G_{d}\left(\boldsymbol{x}, t ; \boldsymbol{x}^{\prime}, t^{\prime}\right) \frac{\partial \boldsymbol{A}\left(\boldsymbol{x}^{\prime}, t^{\prime}\right)}{\partial t^{\prime}} \mathrm{d} V^{\prime} \mathrm{d} t^{\prime} .
\end{aligned}
$$

We can write the last term as:

$$
\frac{1}{4 \pi c} \frac{\partial}{\partial t} \int G_{d}\left(\boldsymbol{x}, t ; \boldsymbol{x}^{\prime}, t^{\prime}\right)\left(\boldsymbol{E}\left(\boldsymbol{x}^{\prime}, t^{\prime}\right)+\nabla^{\prime} \varphi\left(\boldsymbol{x}^{\prime}, t^{\prime}\right)\right) \mathrm{d} V^{\prime} \mathrm{d} t^{\prime} .
$$

So we obtain coincidence between both decompositions. So, any gauge transformation to the Lorenz gauge is clearly equivalent to a hyperbolic Helmholtz decomposition. And, as dictated by theorem 7 any hyperbolic Helmholtz decomposition of the potential is a gauge transformation to the Lorenz gauge.

Theorem 8: Any gauge transformation to the parabolic gauge of a time dependent $S^{k}$ vector potential field $\boldsymbol{A}$ in any gauge or no gauge at all, is a parabolic Helmholtz decomposition if the following conditions are satisfied:

1) $\gamma_{P} \in S^{k}, k>1$

2) $\boldsymbol{A}_{p}$ the potential in the parabolic gauge is $S^{k}$ where $\gamma_{P}$ is the gauge.

Proof: because we lack a nice space-time geometry for the parabolic gauge, we start from the gauge transformation:

$$
\begin{gathered}
A=\boldsymbol{A}_{P}+\nabla \gamma_{P} \\
\varphi=\varphi_{P}-\frac{1}{C} \frac{\partial \gamma_{P}}{\partial t} .
\end{gathered}
$$

From here we write down: 


$$
\nabla \times \nabla \times \boldsymbol{A}=-\frac{4 \pi D}{c} \nabla \varphi_{P}-\Delta \boldsymbol{A}_{P}
$$

Hence:

$$
\nabla \times \nabla \times \boldsymbol{A}=\frac{4 \pi D}{c}\left(\boldsymbol{E}+\frac{1}{c} \frac{\partial \boldsymbol{A}_{P}}{\partial t}\right)-\Delta \boldsymbol{A}_{P}
$$

So:

$$
\nabla \times \nabla \times \boldsymbol{A}-\frac{4 \pi D}{c} \boldsymbol{E}=-\left(\Delta-\frac{4 \pi D}{c^{2}} \frac{\partial}{\partial t}\right) \boldsymbol{A}_{P} .
$$

Now we get from (51) using (52):

$$
\begin{gathered}
\nabla \cdot \boldsymbol{A}=-\frac{4 \pi D}{c} \varphi_{P}+\Delta \gamma_{L} \\
\nabla \cdot \boldsymbol{A}=-\frac{4 \pi D}{c} \varphi-\frac{4 \pi D}{c^{2}} \frac{\partial \gamma_{L}}{\partial t}+\Delta \gamma_{L} .
\end{gathered}
$$

So:

$$
\nabla \cdot \mathrm{A}+\frac{4 \pi D}{c} \varphi=-\left(\Delta-\frac{4 \pi D}{c^{2}} \frac{\partial}{\partial t}\right) \gamma_{L}
$$

From the Equations ((53), (54)) we obtain the parabolic Helmholtz decomposition following the same steps that in the previous theorem. QED

Theorem 9: A parabolic Helmholtz decomposition of the $S^{k}$ time dependent vector potential $\boldsymbol{A}$ and the $S^{k}$ current density $\mathbf{J}$ in Euclidean 3-space that satisfy Maxwell equations is, locally, a gauge transformation to the parabolic gauge that induces the corresponding gauge transformation of the $S^{k}$ scalar potential.

Proof: just like before we made a parabolic Helmholtz decomposition of the vector potential:

$$
\boldsymbol{A}=\boldsymbol{A}_{P}+\boldsymbol{A}_{i},
$$

where $\nabla \times A_{i}=0, \nabla \cdot A_{P}(x, t)=-\frac{4 \pi D}{c} \varphi_{P}(x, t)$, so Equations ((17), (18)) become:

$$
\begin{gathered}
\nabla \times \nabla \times \boldsymbol{A}_{P}=-\frac{1}{c} \frac{\partial}{\partial t} \nabla \varphi-\frac{1}{c^{2}} \frac{\partial^{2} \boldsymbol{A}_{P}}{\partial t^{2}}-\frac{1}{c^{2}} \frac{\partial^{2} \boldsymbol{A}_{i}}{\partial t^{2}}+\frac{4 \pi}{c} \boldsymbol{J} \\
\Delta \varphi+\frac{1}{c} \frac{\partial}{\partial t} \nabla \cdot \boldsymbol{A}_{i}-\frac{4 \pi D}{c} \frac{1}{c} \frac{\partial}{\partial t} \varphi_{P}(\boldsymbol{x}, t)=-4 \pi \varrho .
\end{gathered}
$$

Locally we can write down: $\boldsymbol{A}_{i}=\nabla \gamma_{P}$ so we obtain:

$$
\begin{gathered}
\nabla \times \nabla \times \boldsymbol{A}_{P}=-\frac{1}{c} \frac{\partial}{\partial t} \nabla\left(\varphi+\frac{1}{c} \frac{\partial \gamma_{P}}{\partial t}\right)-\frac{1}{c^{2}} \frac{\partial^{2} \boldsymbol{A}_{P}}{\partial t^{2}}+\frac{4 \pi}{c} \boldsymbol{J} \\
\Delta\left(\varphi+\frac{1}{c} \frac{\partial \gamma_{P}}{\partial t}\right)-\frac{4 \pi D}{c} \frac{1}{c} \frac{\partial}{\partial t} \varphi_{P}(x, t)=-4 \pi \varrho .
\end{gathered}
$$

We see that we can introduce the definition: $\varphi_{P}=\varphi+\frac{1}{C} \frac{\partial \gamma_{P}}{\partial t}$ and using the previous theorem is clear that we have a gauge transformation to the parabolic gauge that satisfies Maxwell equations if, and only if, the equations of the parabolic gauge are satisfied. QED

\section{Models of Maxwell Equations}

All along the paper we have been working the Maxwell's equations in the vacuum (using Gaussian units), i.e. the magnetization and polarization field vectors are zero and quite simply linear constitutive relations ("Max- 
well-Lorentz ether relations”) holds between the electromagnetic field vectors and the field vectors of dielectric displacement and magnetic induction ( i.e. $\boldsymbol{E} \sim \boldsymbol{D}, \boldsymbol{B} \sim \boldsymbol{H}$ ). These hypothesis seems to hold for a vacuum space-time, and we get Maxwell's Equation (2). In this section we shall discuss the reasons underlying our position that a gauge determines a space-time structure. We start by noting that in the Maxwell's equations there are two levels of indetermination. The first level arises out the general covariance of the equations because due to this symmetry under smooth diffeomorphisms there are arbitrary functions in the solutions. The second level is due to typical gauge invariance, which introduces even more arbitrary functions in the function space of solutions. Of course the generally covariant and gauge invariant Maxwell's equations cannot be solved because of its high level of underdetermination that cannot be handled with the usual boundary and initial value problems. So we must fix a lot of structure to get some tractable equations. Now, we can use constitutive relations to fix a space-time, hence, using a gauge condition consistent with the space-time symmetries the whole structure becomes determined. At least in some useful cases. Let's discuss this in more detail.

It is the standpoint of reference [[23], p. 379] that the use of constitutive relations is the reason for the invariance of the equations under the Lorentz group. However in [24] it is proved that Maxwell's equations are covariant under the group of space-time $C^{k}$ diffeomorphisms (see [25] for a discussion of the thesis that all equations of physics are generally covariant). So, using Post conventions (like the use of m.k.s. units), the covariant Maxwell's equations are:

$$
\begin{aligned}
& \partial_{[\mu} F_{\rho \sigma]}=0 \\
& \partial_{\mu} G^{\rho \mu}=j^{\rho}
\end{aligned}
$$

where $F_{\rho \sigma}$ is the electromagnetic field tensor, $G^{\rho \mu}$ is the tensor containing the dielectric displacement and the magnetic induction vectors, $j^{\rho}$ is the four current and the symbol [] means antisymmetrization of the enclosed indexes. As is well known (60) is equivalent to $\partial_{\mu} * F^{\rho \mu}=0$ (see, e.g. [26] p. 557) where

$* F^{\rho \mu}=-\frac{1}{2} \epsilon_{\rho \mu \sigma \tau} F_{\sigma \tau}$ is the dual of the electromagnetic field tensor. The constitutive relations are also generally covariant relations when we write them in the form ([24] p. 127):

$$
G^{\rho \mu}=\frac{1}{2} \gamma^{\rho \mu \sigma \tau} F_{\sigma \tau}
$$

For a non-polarizable and non-magnetizable medium, like a vacuum space-time. So, the Equations ((60)-(62)) are generally covariant and independent of any metric or space-time form features. But they are highly underdetermined. The matrix $\gamma^{\rho \mu \sigma \tau}$ contains all the information about the properties of the medium, so, clearly, all the information about the geometry involved in the distribution in space-time of the electromagnetic field. In order to begin to fix the structure we made the assumptions that our vacuum space-time is uniform, isotropic, non-dispersive and linear, therefore we propose (following Post op. cit. p. 179 using a Minkowski space-time rather than a curved space-time):

$$
\gamma^{\rho \mu \sigma \tau}=B\left(\eta^{\rho \sigma} \eta^{\mu \tau}-\eta^{\rho \tau} \eta^{\mu \sigma}\right)
$$

with $\eta_{\mu \tau}$ the Minkowski metric and $B$ a coupling constant dimensionally consistent, so we obtain the following Maxwell equations (using Post conventions instead of those of the previous section):

$$
\begin{gathered}
F_{\mu \sigma}=2 \partial_{[\mu} A_{\sigma]} \\
\partial_{\mu} \partial^{\rho} A^{\mu}-\partial_{\sigma} \partial^{\sigma} A^{\rho}=\frac{1}{B} j^{\rho}
\end{gathered}
$$

Equation (65) are naturally Lorentz invariant, i.e. invariant under Lorentz transformations only because the relation (63) is Lorentz invariant only. Of course the Equation (64) is generally covariant by itself, but this symmetry is broken because we must consider the whole set of Equation (63) that lead us to (65). So we have seen now how the Lorentz covariance is imposed on the field equations and how the space-time form is defined using the symmetry properties of the differential equations that are derived directly from (63). But the Equations ((64), (65)) possess another symmetry, again an infinite-dimensional one: the gauge symmetry, hence the boundary value problems are all ill-posed for the Equation (65) yet. In theorem 7, above, we have seen how to use a 
Helmholtz decomposition to eliminate the redundant degrees of freedom, because a Helmholtz decomposition is a gauge transformation to the Lorenz gauge. But following the traditional way we must impose a gauge condition. From the gaze of the accepted doctrine the choice of a gauge condition is conventional, but we can see that we have a methodological principle to follow: we must preserve the Lorentz symmetry of the equations, otherwise our choice of space-time is not consistent with the symmetries of the gauge and again we are breaking the symmetry properties. So, we postulate a Lorenz gauge condition because it is Lorentz invariant. Now the role of the gauge condition is clear and a methodological principle for choosing it is revealed, contrary to the commonly accepted wisdom of conventional choices. Friedman in [25] p. 104 showed how to obtain a set of Maxwell's equations for a Newton-Cartan space-time. Because that space-time involves an absolute space the only symmetries of the theory are spatial rotations and space-time translations. But his way is to define the space-time first and then immerse the equations. Clearly the consistent choice of gauge for that case is the Coulomb gauge because, according to our methodological principle, the gauge must be covariant (or invariant) under spatial rotations and space-time translations. Hence the role of the gauge condition is dismissed in conventional approaches, and our proposal is to start directly from the gauge condition to define the consistent space-time.

In the treatment presented in this section the standard procedure has been illustrated using the example of the Minkowski space-time, and we have seen that the choice of gauge comes at the end, but we can reverse things and fix the gauge at the beginning, so that what we must choose is a space-time consistent with the symmetries of the equations in order to fix the whole space of solutions. Hence we have the following methodological principles:

PA) If we determine the Maxwell's equations by fixing the space-time first, hence the choice of gauge condition must respect the space-time symmetry group.

PB) If we determine Maxwell's equations by fixing the gauge first, then the choice of space-time must respect the symmetries of the gauge condition.

It is necessary to discuss yet another point. We have said that the space-time is fixed by means of the symmetries of the differential equations, because given a solution in a, e.g., Lorentz frame of reference, we can obtain another solution in another Lorentz frame using the symmetries, and in this way we define a space-time. This is the path we propose to follow in general: given the gauge we get a set of differential equations whose symmetries determine a space-time. But given that Post proved ([27] p. 188) that a Riemann-Christoffel curvature tensor can be a constitutive tensor if multiplied by the determinant of the metric tensor, so that becomes a density of weight 1 in the contravariant form, but not the other way around; i.e. no all constitutive tensors are curvature tensors; the possibility is open to interpret (63) as a curvature tensor and to establish the space-time form using it, instead of simply using the symmetries of the differential equations. These manifolds, according to Schur's theorem, are manifolds with constant Riemann scalar. If we take a look at [28] p. 91 we can find a local definition (i.e. that holds at a point) of a manifold with constant curvature as one with a curvature tensor of the form (63) with a general metric instead of the Minkowski metric. Note that Schur's theorem (for an elementary proof see [29] p. 323 or [28] p. 93) allow us to make that local definition a global one. Malament comments that all propositions about these manifolds are valid even when the metric tensor is non-positive definite, but the proofs are more involved. Of course (63), if taken as a curvature tensor is the curvature tensor of a manifold with constant Riemann scalar B, so we have not a flat space-time, but, e.g., a Lobachevskian one if B < 0 . In this interpretation the space-time is never Euclidean because the choice B $=0$ give us not any set of Maxwell's equations. So, we believe it better to consider that the space-time is flat, as is usually conceived, instead of consider the Maxwell's equations in spaces of constant curvature, as is suggested if we interpret the constitutive tensor as a curvature tensor.

Hence we have motivated the methodology for the determination of a space-time out of a choice of gauge, so we can proceed to formalize it in a few steps. A nice presentantion of the "problem of space" is available in [30] and [31]

1) The first step lies in the supposition that the space-time is flat, that is, its mathematical representation is going to be made in a 4-dimensional pseudo-riemannian manifold $M$ with zero Riemann curvature tensor. This supposition is independent of Maxwell equations, because these equations by themselves cannot prove that this is the case or not.

2) On our flat manifold $M$ we choose the gauge, which is a relation between the vector potential and the scalar potential defined on $M$. Let us take as examples the gauges we have worked in the previous sections: the elliptic, the parabolic and the hyperbolic gauges. 
3) Once we have the gauge we can define a set of field equations for the potentials defined on $M$. From the gauges, we are working the field equations which are elliptic, hyperbolic and parabolic inhomogeneous equations.

4) We now search the symmetries of the field equations using the following procedure:

4.1) We calculate the Lie point symmetries of the differential operator involved, i.e. if $O$ is an operator of the elliptic, parabolic or hyperbolic type we calculate the Lie point symmetry group of the equation: $O f=0$. In this way we obtain a representation on the 4-dimensional manifold $M$ of a continuous group of transformations of $n$ parameters $\epsilon_{1}, \cdots, \epsilon_{n}$ which we shall denote by $G_{O}$. This group is represented on $M$ by the equations:

$$
\bar{x}_{\sigma}=\bar{x}_{\sigma}\left(x_{\sigma}, \epsilon_{1}, \cdots, \epsilon_{n}\right), \sigma=0,1,2,3 \text {. }
$$

That satisfies all the conditions of a Lie group of transformations. As usual, 0 is for time.

4.2) Once we have the group $G_{O}$ acting on the space-time $M$ and leaving the differential operator $O$ invariant, we must induce a linear representation of this group on the functional space of solutions of the field equations, i.e.

$$
\bar{A}_{\sigma}=\sum \Omega_{\sigma \mu}\left(\epsilon_{1}, \cdots, \epsilon_{n}\right) A_{\mu} .
$$

where, as usual, $A_{0}$ is the scalar potential and $\Omega_{\sigma \mu}$ the coefficients of the induced group representation depending on the $n$ group parameters but not on the space-time points. This same transformation rule must be followed by the matter fields - the current and the density.

5) Directly from the Lie algebra $g_{O}$ we calculate the metric $g$ that the group leaves invariant. This can be made by means of Killing's equation $L_{X} g=0, X \in g_{O}$ with $L_{X}$ the Lie derivative of 2-tensors, or we can proceed as follows: we search for a scalar group invariant with all the properties of a metric, because sometimes Killing equations are underdetermined.

So, following the previous steps, we can define a space-time form out of the gauge. But the procedure is reversible, because we can define a group of symmetries out of physical arguments, then a metric and finally, a set of equations invariant under the group representations. Historically this was the way used by Einstein to define the space-time symmetries of STR and the normal procedure in quantum field theory: given the Lorentz group we may construct all the invariant wave equations, see, e.g., [32], where the authors tell us (p. 212) that the representations of the Lorentz group may replace the wave equation. In our case the gauge defines the field equations, hence we must construct the group. With this background we believe that the following definition has been motivated.

Definition: We say that $T_{M}$ is a model of Maxwell equations' solutions, if and only if, the following objects $M, g, \boldsymbol{A}, \varphi, G, N(\boldsymbol{A}, \varphi), F_{1}(\boldsymbol{A}, \varphi), F_{2}(\boldsymbol{A}, \varphi), R$ exist such that:

1) $T_{M}=\left\langle M, g, \boldsymbol{A}, \varphi, G, N(\boldsymbol{A}, \varphi), F_{1}(\boldsymbol{A}, \varphi), F_{2}(\boldsymbol{A}, \varphi), R\right\rangle$;

2) $M$ is a 4-dimensional pseudo-riemannian manifold;

3) $g$ is a pseudo-metric defined on $T M \times T M$;

4) $\boldsymbol{A}: M \rightarrow \mathbf{R}^{3}, \varphi: M \rightarrow \mathbf{R}$ are potential of the Maxwell equations;

5) $N(\boldsymbol{A}, \varphi)=0$ is the gauge involving $\boldsymbol{A}, \varphi$ and its derivatives, and preserved by all allowed gauge transformations;

6) $F_{1}(\boldsymbol{A}, \varphi)=0, \quad F_{2}(\boldsymbol{A}, \varphi)=0$ are the field Equations ((17), (18)) satisfied by the potentials;

7) $G$ is the symmetry group of the metric tensor $g$ and the field equations defined by the gauge $N(\boldsymbol{A}, \varphi)=0$

8) $R$ is the Riemann curvature tensor such that $R=0$, so this equation is an independent field equation determining the flatness of $M$.

So, in the terminology of [27] (see also [25] the discussion on pages 46-70) we have a local space-time theory. With this definition, and the procedure that we have outlined, we can say that a gauge defines a model of Maxwell equations' solutions, so a form for space-time. The models of Maxwell equations defined by a gauge may be related by a gauge transformation, so, and this is the common wisdom, all of them are mathematically equivalent. But, and this is our point, they are not empirically equivalent because they imply very different observational consequences. This can be seen with the example of the Coulomb gauge and the Lorenz gauge. If we use the Coulomb gauge we can define an electrodynamics that accept as symmetry group the Galilean group. We must just take a look at the equations (S) to see why this is the case: 


$$
\begin{aligned}
\Delta \varphi_{C} & =-4 \pi \varrho \\
\Delta \boldsymbol{A}_{C} & =\frac{1}{c} \frac{\partial \boldsymbol{E}}{\partial t}+\frac{4 \pi}{c} \boldsymbol{J}
\end{aligned}
$$

the Laplace operator admits as symmetry group the Galilean group, i.e., it is an invariant operator for space translations, time translations, space rotations and Galilean boosts, so, we can induce a representation of this group to make that the scalar and vector potentials and the charge and current densities transform adequately under this representation. The transformation rules are as follows, if $g_{V}$ is a Galilean boost we have:

$$
\begin{aligned}
& g_{v}^{*} \boldsymbol{A}_{C}(\boldsymbol{x}, t)=\boldsymbol{A}_{C}^{\prime}\left(\boldsymbol{x}^{\prime}, t^{\prime}\right)=\boldsymbol{A}_{C}(\boldsymbol{x}-\boldsymbol{v} t, t) \\
& g_{v}^{*} \varphi_{C}(\boldsymbol{x}, t)=\varphi_{C}^{\prime}\left(\boldsymbol{x}^{\prime}, t^{\prime}\right)=\varphi_{C}(\boldsymbol{x}-\boldsymbol{v} t, t) \\
& g_{v}^{*} \boldsymbol{E}(\boldsymbol{x}, t)=\boldsymbol{E}^{\prime}\left(\boldsymbol{x}^{\prime}, t^{\prime}\right)=\boldsymbol{E}(\boldsymbol{x}-\boldsymbol{v} t, t) \\
& g_{v}^{*} \boldsymbol{J}(\boldsymbol{x}, t)=\boldsymbol{J}^{\prime}\left(\boldsymbol{x}^{\prime}, t^{\prime}\right)+\frac{1}{4 \pi}\left(\boldsymbol{v} \cdot \nabla^{\prime}\right) \boldsymbol{E}^{\prime} \\
& g_{v}^{*} \varrho(\boldsymbol{x}, t)=\varrho^{\prime}\left(\boldsymbol{x}^{\prime}, t^{\prime}\right)=\varrho(\boldsymbol{x}-\boldsymbol{v} t, t)
\end{aligned}
$$

Therefore the induced representation of the Galilean group turns out to be the trivial one, only the current density requires a nontrivial transformation rule. And, as can be seen, charge conservation is preserved in all Galilean frames. As usual the primes indicate the moving inertial frame. With these rules the equations (S) are invariant under the action of the Galilean group. In fact, the only difficult term is Maxwell's displacement current $\frac{\partial \boldsymbol{E}}{\partial t}$ which under a Galilean boost becomes: $\frac{\partial \boldsymbol{E}^{\prime}}{\partial t^{\prime}}-\left(\boldsymbol{v} \cdot \nabla^{\prime}\right) \boldsymbol{E}^{\prime}$ this is the reason for the transformation law for the current density. So the usefulness of the Stewart's representation lies in this easy proof of invariance.

Of course, if we use the Galilean group we can define a Newtonian space-time (see e.g. [25] chapter 3 and [31] chapter 4) which admits the following description. On our manifold $M$ we can define 3-spaces of simultaneity:

$$
S P_{t}=\left\{x, y, z, t \in \mathbf{R}^{4} \mid t=\text { constant }\right\} .
$$

So if our Euclidean space-time is the 4-dimensional manifold $M$ we can write down: $M=\bigcup_{t \in J} S P_{t}, J \subset \mathbf{R}$, which we can read as a global foliation of $M$ by simultaneity 3-spaces, hence, on each one of the leaves we can obtain an instantaneous solution, existing on all its points at each time slice. In this way we obtain absolute time and Galilean-relative space. In this way we have constructed a Galilean invariant electrodynamics compatible with a Newtonian space-time, without the use of the Inonu-Wigner contraction, i.e., taking the limit of infinite velocity. The Helmholtz decomposition (or the Coulomb gauge) include this information into the space of solutions of the Maxwell equations. However, we know since the inception of the STR that space-time cannot be globally Euclidean because we cannot define a global relation of simultaneity, but this information is not contained in the Coulomb gauge, but in the Lorenz gauge. Therefore we require, if we want to accommodate into our physics the fact that there is a limit to the velocity of light, to change the gauge and erect another space-time. The doctrine of gauge transformations tells us that this we can do, but if we do so, we are constructing a new model of Maxwell equations that is not empirically equivalent to the previous one (in this case the Coulomb gauge). So we cannot choose the gauge by taste, such a choice imply empirical consequences. Now we know why in the Coulomb gauge, instantaneous solutions appear: they are a manifestation of the underlying Newtonian space-time and of the assumptions of absolute time and Galilean-relative space. So, to obtain a set of Maxwell equations compatible with Newtonian mechanics we just need to choose a gauge.

\section{Conclusions}

In all our journey, we have find little role for the gauge transformations, and specially for the doctrine that all gauges are equivalent. To tackle the question we introduce a distinction:

- We say that the gauge condition presents "local empirical irrelevance" because at the level of direct human experience it is irrelevant the global space-time form. This is the "reason" for the plausibility of the doctrine that the gauge conditions are selected by taste or any pragmatically oriented criterion. 
- We say that the gauge condition presents "global empirical significance” because at the level of our speculations of the global form of the space-time and the nature of the universe; subjects far away from direct human experience; the gauge condition is determined by the space-time structure. In a theory of everything the gauge condition is not a matter of convention, but of fact and logically constructed theory.

So, because of its “local empirical irrelevance”, we can use any gauge for solving the Maxwell's equations for matters of direct human experience, like the development of telecommunications or any other technological development directly related to human life. But we believe that the gauge is not a question of taste, but of direct observation and is related to the facts that determine the global form of space-time.

Let us try to resume the paper in a few propositions.

1) At the level of the electromagnetic field strengths, a Helmholtz decomposition is the equivalent of a gauge transformation at the level of the potentials. So, like the gauge transformations, there are many Helmholtz decompositions that depend on the differential operator used to perform the decomposition. In particular, in this paper we have proved that for a linear differential operator of the second order $O$ in 4 variables there are three natural Helmholtz decompositions according to the three canonical forms of $O$.

2) We have shown that each Helmholtz decomposition corresponds to a gauge transformation.

3) We have outlined the method to erect a space-time out of a gauge (or a Helmholtz decomposition).

4) The Aharonov-Bohm experiment suggests that the potentials may have a physical meaning, so the criteria of gauge invariance is not necessary because there are objects (the potentials) that are not gauge invariant but have observable consequences. The proof that the Coulomb gauge potentials are gauge invariant seems to suggest that gauge invariance is not sufficient criteria of physical relevance because there are gauge invariant quantities that being gauge invariant, are not physically relevant. In this paper we propose that a choice of gauge defines a space-time structure, so, its relevance is empirical, not just theoretical. The Coulomb potentials may be relevant in conditions where a Newtonian space-time underlies the phenomena, but globally these potentials are not physically relevant.

5) A Helmholtz decomposition is as valid as a gauge, so all the solutions obtained using one are as correct as all the solutions using a gauge.

6) What we must remember from time to time is not that all the gauges are mathematically equivalent, but that the choice of gauge implies physical consequences, so, different physics, a position that we have justified at length in Section 5.

\section{Acknowledgements}

RAF acknowledges the kindness of K. H. Yang for sending him a copy of ref [19] with the methodological remark that one must consider, carefully, the explicit solutions of the equations involved.

\section{References}

[1] Heras, J. (2003) American Journal of Physics, 71, 729-730. http://dx.doi.org/10.1119/1.1555894

[2] Woodside, D.A. (2009) American Journal of Physics, 77, 438-446. http://dx.doi.org/10.1119/1.3076300

[3] Woodside, D.A. (1999) Journal of Mathematical Physics, 40, 4911-4943. http://dx.doi.org/10.1063/1.533007

[4] Baker, B.B. and Copson, E.T. (1950) The Mathematical Theory of Huygens' Principle. Clarendon Press, Oxford.

[5] Rohrlich, F. (2002) American Journal of Physics, 70, 411-414. http://dx.doi.org/10.1119/1.1435345

[6] Dmitriyev, V.P. (2004) European Journal of Physics, 25, L23-L27.

[7] Heras, J. (1994) American Journal of Physics, 62, 525-531. http://dx.doi.org/10.1119/1.17512

[8] McQuistan, R.B. (1965) Scalar and Vector Fields: A Physical Interpretation. Wiley, New York.

[9] Heras, J. (1996) American Journal of Physics, 64, 409-413. http://dx.doi.org/10.1119/1.18255

[10] Rohrlich, F. (1965) Classical Charged Particles. Addison-Wesley, Boston, 68.

[11] Heras, J. (1994) American Journal of Physics, 62, 1109-1115. http://dx.doi.org/10.1119/1.17759

[12] Heras, J. (1995) American Journal of Physics, 63, 928-932. http://dx.doi.org/10.1119/1.18086

[13] Davis, A. (2006) American Journal of Physics, 74, 72-76. http://dx.doi.org/10.1119/1.2121756

[14] Heras, J. (2006) American Journal of Physics, 74, 744-745. http://dx.doi.org/10.1119/1.2206574

[15] Heras, J. (1990) American Journal of Physics, 58, 153-154. http://dx.doi.org/10.1119/1.16225 
[16] Belot, G. (1998) British Journal for the Philosophy of Science, 49, 531-555. http://dx.doi.org/10.1093/bjps/49.4.531

[17] Jackson, J.D. (2002) American Journal of Physics, 70, 917-928. http://dx.doi.org/10.1119/1.1491265

[18] Heras, J. (2007) American Journal of Physics, 75, 176-186. http://dx.doi.org/10.1119/1.2400238

[19] Yang, K.H. (2005) American Journal of Physics, 73, 742-751. http://dx.doi.org/10.1119/1.1938949

[20] Chubykalo, A., Espinoza, A. and Alvarado, F.R. (2011) Physica Scripta, 84, 015009.

[21] Engelhardt, W. and Onoochin, V. (2012) Physica Scripta, 85, 047001.

[22] Stewart, A.M. (2003) European Journal of Physics, 24, 519-524. http://dx.doi.org/10.1088/0143-0807/24/5/308

[23] Bargmann, V. and Wigner, E.P. (1948) Proceedings of the National Academy of Sciences of the United States of America, 34, 211-223. http://dx.doi.org/10.1073/pnas.34.5.211

[24] Post, E.J. (1962) Formal Structure of Electromagnetics. North-Holland, Amsterdam.

[25] Friedman, M. (1983) Foundations of Space-Time Theories. Princeton University Press, Princeton.

[26] Jackson, J.D. (1999) Classical Electrodynamics. Wiley, New York.

[27] Earman, J. and Norton, J.D. (1987) British Journal for the Philosophy of Science, 38, 515-525. http://dx.doi.org/10.1093/bjps/38.4.515

[28] Malament, D. (2012) Topics in the Foundations of General Relativity and Newtonian Gravitation Theory. University of Chicago Press, Chicago. http://dx.doi.org/10.7208/chicago/9780226502472.001.0001

[29] Lass, H. (1950) Vector and Tensor Analysis. McGraw-Hill, New York.

[30] Bernard, J. (2015) Studies in History and Philosophy of Modern Physics, 52, 251-266.

[31] Merker, J. (2010) Le problème de l'espace: Sophus Lie, Friedrich Engel et le problême de Riemann-Helmholtz. Hermann, Paris.

[32] Edelen, D.G.B. (1985) Applied Exterior Calculus. Wiley, New York. 